\begin{abstract}
Zusammenfassung
In diesem Bericht werden die Epiphysenfrakturen zuerst allgemein besprochen. Dabei werden die anatomischen Besonderheiten, die zur Entwicklung der Salter-Harris-Klassifikation führten, diskutiert. Die möglichen Epiphysenfrakturen werden dann anhand der einzelnen Knochen durchgesprochen und ihre Behandlungsarten diskutiert.

Allgemein kann gesagt werden, daß Epiphysenfrakturen bei wachsenden Fohlen in vielen Fällen erfolgreich behandelt werden können, doch muß eine vorsichtige Prognose für einen späteren Einsatz als Gebrauchspferde gestellt werden.

Für den Transport in eine Klinik sollten Fohlen mit einer Epiphysenfraktur unter allen Umständen mit einer Schiene oder einem Fiberglasverband temporär fixiert werden.

Epiphysenfrakturen der distalen Gliedmaßen können unter Umständen konservativ mit Fiberglasverbänden behandelt werden, doch ist eine chirurgische Versorgung der Fraktur in den meisten Fällen vorzuziehen. Dabei ist es notwendig, die Grundsatzprinzipien der Osteosynthese einzuhalten.

Die Implantate sollten so früh wie möglich entfernt werden, um das normale Wachstum so wenig wie möglich zu beeinträchtigen. Die Behandlung der Frakturen sollte so schnell wie möglich erfolgen, um einer Zerstörung der Wachstumsplatte oder des Knochens an der Frakturstelle vorzubeugen.

Wachstumsstörungen sind die am häufigsten auftretenden Komplikationen bei Epiphysenfrakturen. Daneben treten aber auch Infektionen, Arthrosen und Zusammenbrüche der Osteosynthese auf.
\end{abstract}

\section{Fractures in the Growing Foal Part I:}

Epiphyseal Fractures

This paper discusses general considerations for epiphyseal fractures and the anatomical differences which led to the Salter-Harris-classification are explained. The various locations and fracture configurations in the different bones are mentioned, and suggestions for their treatment are made.

Epiphyseal fractures in growing foals have generally a favourable prognosis for healing, if treated properly. However, the prognosis for future use as an athlete has to be judged as guarded.

The limb with an epiphyseal fracture should under any circumstances be splinted or cast for the transport to hospital.

Epiphyseal fractures of the distal portions of the limb may be treated by cast application in some cases. More frequently, however, surgical reduction followed by some form of internal fixation is preferred. It is important to follow the basic principles of internal fixation.

The implants should be removed at the earliest convenience, to prevent undue growth disturbances. Epiphyseal fractures should be treated as soon as possible to avoid further destruction of the growth plate through continuous movement at the fracture site. Growth disturbances are the most frequently encountered complications with epiphyseal fractures. Other complications include infection, osteomyelitis, degenerative joint disease and breakdown of the fracture fixation.

und mit Knochengewebe ersetzt werden. Nach Abschluß dieser Vorgänge obliteriert der Epiphysenknorpel, und der Knochen präsentiert sich als eine Einheit. Dieser sogenannte Epiphysenschluß vollzieht sich in den verschiedenen Knochen zu verschiedenen Zeitpunkten.

Die Epiphysen der Röhrenknochen sind dauerndem Druck durch das anliegende Gelenk ausgesetzt und werden daher als „Druckepiphysen“ bezeichnet (Salter und Harris, 1963). Epiphysenfugen im Zusammenhang mit Druckepiphysen sind ausschließlich für das Längenwachstum durch endochondrale Ossifikation verantwortlich (Braden, 1981). Andere Knochen, z. B. die Ulna, besitzen eine „Zugepiphyse“, 


\begin{tabular}{|c|c|c|c|}
\hline Knochen & $\begin{array}{l}\text { Totale Anzahl } \\
\text { Frakturen } \\
\text { Texas A\&M }\end{array}$ & $\begin{array}{l}\text { Epiphyser } \\
\text { Texas A\&M }\end{array}$ & $\begin{array}{l}\text { nfrakturen } \\
\text { Embertson et } \\
\text { al. }(1986 \text { a) }\end{array}$ \\
\hline Scapula & 22 & 9 & 5 \\
\hline $\begin{array}{l}\text { Humerus } \\
\text { Proximale Epiphyse } \\
\text { Tuberculum majus } \\
\text { Medialer Epicondylus } \\
\text { Distale Epiphyse }\end{array}$ & 40 & $\begin{array}{l}6 \\
(4) \\
(1) \\
(1) \\
(0)\end{array}$ & $\begin{array}{l}11 \\
(1) \\
(1) \\
(2) \\
(7)\end{array}$ \\
\hline $\begin{array}{l}\text { Radius } \\
\text { Proximale Epiphyse } \\
\text { Distale Epiphyse }\end{array}$ & 35 & $\begin{array}{l}8 \\
(7) \\
(1)\end{array}$ & $\begin{array}{l}7 \\
(3) \\
(4)\end{array}$ \\
\hline Ulna & 61 & 22 & 10 \\
\hline $\begin{array}{l}\text { McllI/MtIII } \\
\text { Mclll } \\
\text { Mtlll }\end{array}$ & 65 & $\begin{array}{c}3 \\
(1) \\
(2)\end{array}$ & $\begin{array}{l}7 \\
(3) \\
(4)\end{array}$ \\
\hline Proximale Phalanx & 38 & 3 & 1 \\
\hline $\begin{array}{l}\text { Femur } \\
\text { Proximale Epiphyse } \\
\text { Trochanter major } \\
\text { Distale Epiphyse }\end{array}$ & 59 & $\begin{array}{c}29 \\
(15) \\
(0) \\
(14)\end{array}$ & $\begin{array}{l}21 \\
(11) \\
(3) \\
(7)\end{array}$ \\
\hline $\begin{array}{l}\text { Tibia } \\
\text { Proximale Epiphyse } \\
\text { Crista tibialis } \\
\text { Distale Epiphyse }\end{array}$ & 55 & $\begin{array}{c}16 \\
(11) \\
(1) \\
(4)\end{array}$ & $\begin{array}{l}8 \\
(3) \\
(1) \\
(4)\end{array}$ \\
\hline Total & 421 & $96(23 \%)$ & $70(21 \%)$ \\
\hline
\end{tabular}

Tab. 1: Anzahl der Epiphysenfrakturen und deren Anteil an allen Röhrenbeinfrakturen, die seit 1978 in die Texas-A\& M-Universitätsklinik eingeliefert wurden. Diese Frakturen werden mit den Daten von Embertson et al. (1986a) verglichen. () Unterteilung der verschiedenen Epiphysenfrakturen im gleichen Knochen.

deren Wachstumsplatte aber nicht am Längenwachstum des Knochens beteiligt ist und keine Gelenksoberfläche besitzt (Salter und Harris, 1963).

Kenntnisse über die mikroskopische Anatomie der Epiphysenfuge sind unerläßlich, um die Auswirkungen von Epiphysenregionstraumen zu verstehen. Die Knorpelfuge ist zwischen die Epiphyse und Metaphyse eingebettet und vom Perichondrium umgeben (Abb. 1). Unter dem Mikroskop kann von der Epiphyse in Richtung Metaphyse die Zone des ruhenden Knorpels, gefolgt von der Proliferations-, Maturations- und Kalzifikationszone, unterschieden werden (Ham und Cormack, 1979).

Die ruhende Knorpelzone beteiligt sich nicht am Längenwachstum, doch ist diese Zone für die Verankerung der Knorpelfuge an der Epiphyse verantwortlich. Afferente Blutgefäße dringen von der Epiphyse her in die ruhende Knorpelschicht ein und ernähren die Zellen der Knorpelfuge durch Diffusion (Ham und Cormack, 1979). Die Proliferationszone produziert neue Knorpelzellen, welche sich kolonnenartig einreihen und langsam hypertrophieren. Der Übergang von der Proliferationszone in die Maturationszone geht mit zunehmendem Wachstum der Zelle gleitend vor sich. Diese beiden Zonen sind für das Längenwachstum verantwortlich. In der Kalzifikationszone wird zunächst die Interzellularsubstanz von metaphysären Blutkapillaren infiltriert und kalzifiziert. Osteoblasten, die sich in dieser Zone ansiedeln, deponieren eine Knochenschicht über das netzartige Knorpelmatrixgeflecht. Mit zunehmender Distanz von den Basiszonen der Epiphysenfuge nimmt die Zellernährung mittels Diffusion ab, und die Knorpelzellen degenerieren (Ham und Cormack, 1979).
Die Interzellularsubstanz ist für die Resistenz und Widerstandsfähigkeit der Epiphysenfuge verantwortlich - und nicht die eigentlichen Zellen (Salter und Harris, 1963). Diese Matrix besteht aus Kollagenfasern, welche longitudinal orientiert und in Zementsubstanz eingebettet sind (Ham und Cormack, 1979). In den Basiszonen der Epiphysenfuge, z. B. in der Zellproliferationszone, ist die Matrix in großer Menge vorhanden, was die Widerstandsfähigkeit erheblich vergrößert. Die Interzellularsubstanz verringert sich im gleichen $\mathrm{Maße}$, wie die Zellen hypertrophieren, was eine zunehmende Schwächung der Epiphysenfuge zur Folge hat (Salter und Harris, 1963; Weber, 1980; Brashear, 1959).

Der relativen Schwäche wegen wird die Epiphysenfuge als das schwächste Glied des appendikulären Skeletts betrachtet (Salter und Harris, 1963; Tachidjian, 1972). Obwohl Knorpelgewebe schwächer als Knochen ist, werden relativ selten Frakturen beobachtet, die sich rein auf die Epiphysenfuge konzentrieren. In den meisten Fällen ist auch der umliegende Knochen mindestens teilweise betroffen ( $\mathrm{Sal}$ ter, 1980). Das äußerst widerstandsfähige Perichondrium, das die Epiphysenfuge umgibt, kann Biegungs-, Torsionsund Kompressionskräften gut widerstehen, ist aber gegenüber Scherkräften, die für das Entstehen von Epiphysenfrakturen verantwortlich sind, relativ widerstandslos (Ham und Cormack, 1979).

Die unbeeinträchtigte Blutzufuhr zur Epiphysenfuge ist für ein normales Knochenwachstum unerläßlich. Die afferenten Gefäße, welche die Epiphyse ernähren, dringen von der Oberfläche her ein. Die Unterbrechung der Blutzufuhr zur Epiphyse kann sich in ischämischer Nekrose der Epiphyse und Epiphysenfuge ausdrücken, da - wie erwähnt -

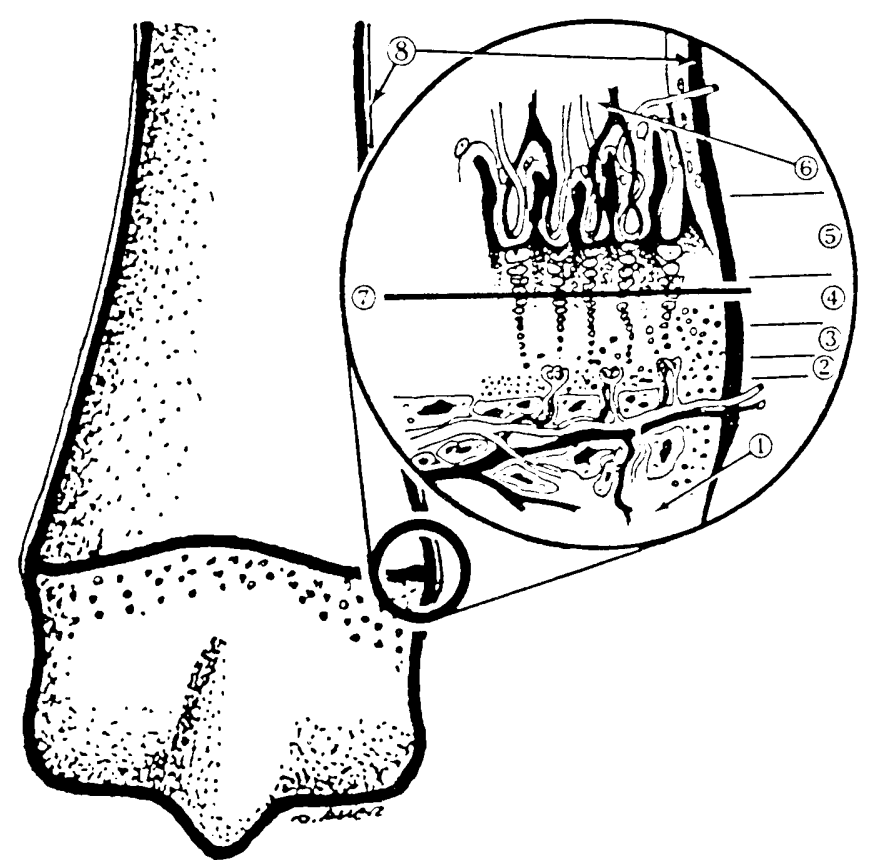

Abb. 1: Skizze der distalen Epiphysenregion eines Metakarpus mit einer Detailzeichnung als Einlage. (1) Epiphyse, (2) Zone des ruhenden Knorpels, von Blutkapillaren durchdrungen, (3) Proliferationszone, (4) Maturationszone, (5) Degenerationszone, (6) Metaphyse mit nutritiven Blutgefäßen, (7) wahrscheinliche Frakturebene, (8) Periost. 
die Basiszonen der Knorpelfugen von epiphysären Gefäßen ernährt werden. Dies wirkt sich in permanenter Wachstumsstörung mit Achsenfehlstellungen und Gelenkaffektionen aus (Weber, 1980). In den meisten Röhrenknochen penetrieren die afferenten Gefäße die Epiphyse in einer gewissen Distanz relativ zur Knorpelfuge (Weber, 1980). Aus diesem Grund werden diese Gefäße selten durch Epiphysenfrakturen beeinträchtigt. In einzelnen anatomischen Spezialfällen traversieren die epiphysealen Gefäße jedoch die Wachstumsplatte, bevor sie die Epiphyse penetrieren (Salter und Harris, 1963). Durch Verletzungen in dieser Gegend können diese Gefäße betroffen werden, was zu den vorher erwähnten Veränderungen führen kann (Salter, 1980). Diese anatomische Konfiguration ist typisch für intraartikuläre Epiphysen, wie z. B. die Epiphyse des Femurkopfes.

Die Blutzufuhr der Ossifikationszone entspringt deren metaphysealen Gefäßen. Verletzungen dieser Gefäße bedingen eine vorübergehende Wachstumsverzögerung (Marretta und Schrader, 1983). Nach chirurgisch induzierter Unterbrechung der diaphysealen Blutzufuhr konnte eine kompensatorische Hyperämie der afferenten Epiphysengefäße nachgewiesen werden (Salter, 1980). Dies resultierte in einem beschleunigten Längenwachstum im Epiphysenbereich. Eine Verletzung der perichondralen Gefäße hat keinen Einfluß auf das Längenwachstum (Weber, 1980). Aus diesen Gründen entscheidet hauptsächlich die An- oder Abwesenheit einer Schädigung der proliferierenden Knorpelzellen über Entwicklung oder Ausbleiben einer Wachstumsstörung. Diese Schädigung kann mechanisch durch direktes Trauma oder Unterbrechung der epiphysären Blutzufuhr entstehen.

\section{Diagnose}

Die meisten Epiphysenprobleme entstehen durch Trauma. Dabei induzieren spezifische Kräfte charakteristische Frakturen (Weber, 1980).

Wenn sich ein Fohlen eine schmerzhafte Verletzung zufügt, die sich durch Schwellung einer Gelenksregion und Nichtbelastung der Gliedmaße kennzeichnet, sollte eine Epiphysenfraktur in Betracht gezogen werden.

Eine genaue Diagnose kann nur mit Hilfe von guten Röntgenaufnahmen erstellt werden. Dabei sollten mindestens 2 Aufnahmen im rechten Winkel zueinander aufgenommen werden. Bestehen anhand dieser Röntgenbilder noch immer Zweifel über eine Frakturkonfiguration, muß durch weitere Aufnahmen in verschiedenen Winkeln die Röntgenuntersuchung ergänzt werden. In Zweifelsfällen können entsprechende Röntgenbilder der gesunden Gliedmaße zu Hilfe genommen werden. Es muß jedoch in Betracht gezogen werden, daß eventuell die vorher verschobenen Frakturfragmente durch Manipulation der Gliedmaße reduziert werden können, was ein annähernd normales Röntgenbild vortäuschen kann.

\section{Klassifikation}

Die Klassifikation von Epiphysenfrakturen anhand verschiedener Frakturtypen wird allgemein als nützlich für
Behandlungsvorschläge und Prognosestellung erachtet. Die Klassifikation nach Salter und Harris (1963) ist heute die generell akzeptierte Form der Einteilung der verschiedenen Epiphysenfrakturen und beschreibt die Art und den Verlauf der Frakturebene im Bereich der Epiphysenfuge.

Diese Prognose anhand des Frakturtyps muß jedoch vorsichtig beurteilt werden, da die röntgenologische Interpretation nicht immer die ganzen anatomischen und physiologischen Veränderungen berücksichtigt. Es muß auch daran erinnert werden, daß auf dem Röntgenbild nur die Fraktur oder die Veränderungen des Knochens dargestellt werden. Der Mechanismus und die Faktoren, die zur Verletzung führten, und die Absorbierung der dabei freigewordenen Energie in den umliegenden Muskeln, Sehnen und Weichteilgeweben sind nicht berücksichtigt. Diese Parameter sind jedoch sehr wichtig für die Prognose.

Salter-Harris-Typus-I-Frakturen bestehen aus einer Separation entlang der ganzen Epiphysenfuge (Abb. 2). Diese Separation vollzieht sich in der Knorpelmaturationszone, welche - wie bereits erwähnt - die schwächste Stelle des Röhrenknochens darstellt. Dennoch sind reine Salter-Harris-Typus-I-Frakturen relativ selten diagnostiziert. Der Grund dafür ist das umliegende Perichondrium, das eine sehr zähe Materie darstellt, die auf Zug und Druck wenig reagiert. Scherkräfte können hingegen eine Ruptur des Perichondriums hervorrufen (Braden, 1981; Weber, 1980). Salter-Harris-Typus-I-Frakturen treten bei Kindern und Kälbern relativ häufig während der Geburt in der proximalen Epiphysenfuge des Femurs auf (Salter und Harris, 1963; Hamilton et al., 1978). Bei Fohlen werden diese Frakturen jedoch in einem Alter von 2 bis 4 Monaten diagnostiziert (Turner et al., 1979), während dieselben Probleme beim Junghund zwischen 3 und 7 Monaten auftreten (Daly,

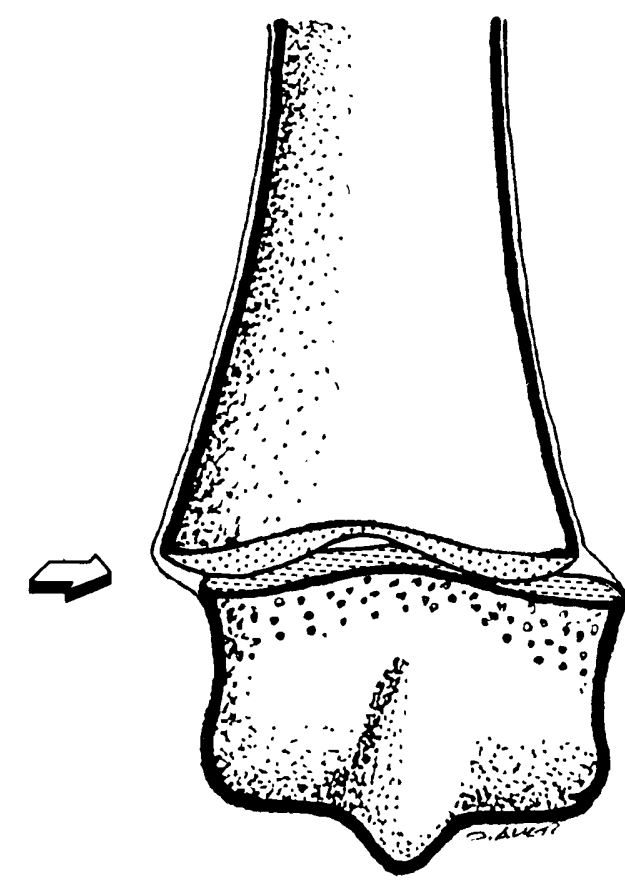

Abb. 2: Skizze einer Salter-Harris-Typus-I-Epiphysenfraktur. Der Pfeil gibt die Kraftrichtung an, welche die Fraktur erzeugte. Das Periost kann intakt bleiben. 


\begin{tabular}{|c|c|c|c|c|c|}
\hline \multirow[t]{2}{*}{ Species } & \multicolumn{4}{|c|}{ Salter-Harris-Frakturtypen } & \multirow{2}{*}{$\begin{array}{l}\text { Andere } \\
\text { Frakturen }\end{array}$} \\
\hline & 1 & $\|$ & III & IV & \\
\hline \multirow{3}{*}{$\begin{array}{l}\text { Pferd } \\
\text { Texas A \& M seit } 1978 \\
\text { Embertson et al. } \\
(1986 \text { a) }\end{array}$} & & & & & \\
\hline & $14 \%$ & $60 \%$ & $14 \%$ & $7 \%$ & $5 \%$ \\
\hline & $13 \%$ & $65 \%$ & $11 \%$ & $11 \%$ & $0 \%$ \\
\hline $\begin{array}{l}\text { Hund } \\
\text { Marretta und } \\
\text { Schrader (1983) }\end{array}$ & $14 \%$ & $51 \%$ & $2 \%$ & $24 \%$ & $9 \%$ \\
\hline \multirow{2}{*}{$\begin{array}{l}\text { Mensch } \\
\text { Rogers (1970) }\end{array}$} & & & & & \\
\hline & $6 \%$ & $75 \%$ & $8 \%$ & $10 \%$ & $1 \%$ \\
\hline
\end{tabular}

Tab. 2: Prozentuale Häufigkeit des Auftretens von den verschiedenen Salter-Harris-Epiphysenfrakturtypen bei Pferd, Hund und Mensch In dieser Aufstellung sind nur Druckepiphysenfrakturen berücksichtigt.

1978). In der Tabelle 2 sind die prozentualen Häufigkeiten, mit welchen die verschiedenen Druckepiphysenfrakturtypen beim Menschen (Rogers, 1970), Hund (Marretta und Schrader, 1983) und Pferd (Embertson et al., 1986a) auftreten, zusammengestellt. Darin wird die Häufigkeit von Salter-Harris-Typus-I-Frakturen mit $6 \%$ beim Menschen, $14 \%$ beim Hund und $13 \%$ beim Pferd angegeben. An unserer Klinik wurden $14 \%$ der Epiphysenfrakturen als Salter-Harris-Typus-I-Frakturen diagnostiziert. Es ist wichtig zu erkennen, daß in den Salter-Harris-Typus-I-Frakturen die Zellschicht des proliferierenden Knorpels intakt und in Verbindung mit der Epiphyse bleibt. Dadurch tritt kein Schaden in der Zellschicht und in der Blutzufuhr der Epiphysenfuge auf (Abb. 1). Deshalb ist auch theoretisch nicht zu erwarten, daß eine Wachstumsstörung auftritt, nachdem diese Frakturen verheilt sind. Das einzige Problem, das in Betracht gezogen werden muß, ist das kontinuierliche Trauma, welches durch das Aneinanderreiben der beiden Frakturenden entsteht. Kann die Fraktur nicht genügend

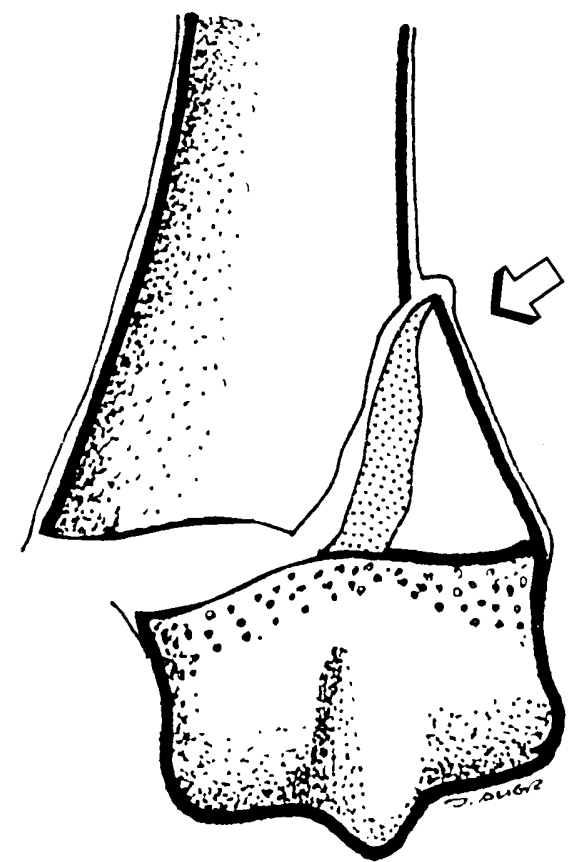

Abb. 3: Skizze einer Salter-Harris-Typus-II-Epiphysenfraktur. Der Pfeil markiert die von schräg oben einwirkende Kraft, die eine Spannung auf der gegenüberliegenden Seite der Epiphysenfuge erzeugte, das Periost zerriß und die Fraktur einleitete. stabilisiert, und Minimalbewegungen zwischen den Frakturenden verhindert werden, so können die tieferen Knorpelschichten der Epiphysenfuge auch zerstört werden. Tritt solch eine Abnützung in Erscheinung, können sich auch in Salter-Harris-Typus-I-Frakturen Wachstumsstörungen entwickeln.

Salter-Harris-Typus-II-Frakturen sind die am häufigsten diagnostizierten Epiphysenfrakturen bei dem Menschen (Salter, 1980), dem Hund, der Katze (Braden, 1981) und auch beim Pferd (Watkins und Auer, 1984; Embertson et al., 1986 a, b). Die Fraktur entsteht entlang der Epiphysenfuge

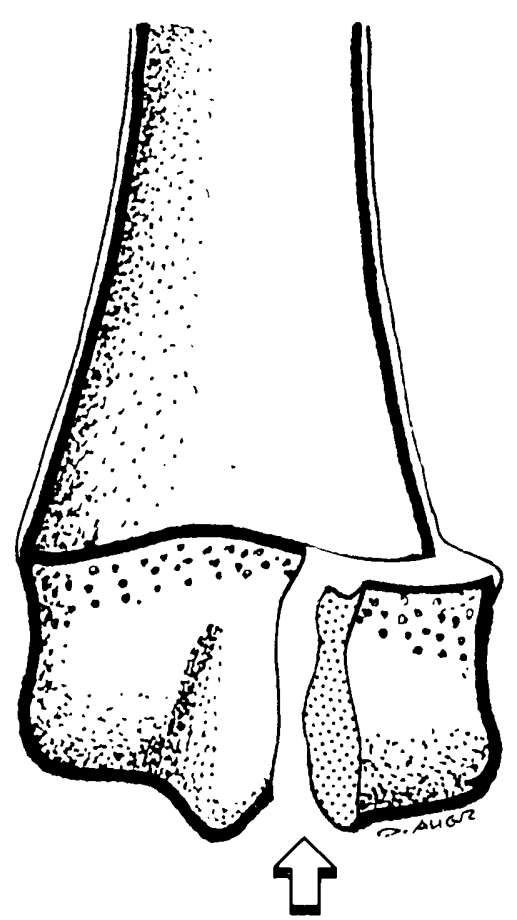

Abb. 4: Skizze einer Salter-Harris-Typus-III-Epiphysenfraktur. Die Krafteinwirkung vcn Richtung des Gelenkes erzeugte eine Fraktur quer durch die Epiphyse

und bricht dann in die Metaphyse ein. Die Salter-HarrisTypus-II-Frakturen entstehen meistens durch schiefe Krafteinwirkung im Bereich der Metaphyse (Abb. 3). Das Perichondrium wird an der konvexen Seite zerrissen, doch bleibt es an der konkaven Seite intakt (Brashear, 1959; Ta. chidjian, 1972). Dieses periosteale Scharnier kann zur Reduktion der Fraktur benützt werden. Wie bei den SalterHarris-Typus-I-Frakturen ist die Prognose in den meisten Fällen gut, da auch bei diesen Frakturen die Zone des proliferierenden Epiphysenknorpels nicht zerstört wird. Daher sollten sich auch keine Wachstumsstörungen entwickeln. Das gleiche Problem wie bei den Typus-I-Frakturen muß auch hier erwähnt werden, und zwar, daß durch ungenügende Stabilisierung der Frakturen eine Zerstörung der Epiphyse auftreten kann.

Der prozentuale Anteil der Salter-Harris-Typus-II-Frakturen gegenüber allen Epiphysenfrakturen wird in Tabelle 2 mit $75 \%$ für den Menschen (Rogers, 1970), 51\% für den Hund (Marretta und Schrader, 1983) und $65 \%$ für das Pferd (Embertson et al., 1986 a) angegeben. Seit 1978 wurden 
$60 \%$ aller Epiphysenfrakturen an der Texas-A\&M-Universitätsklinik als Salter-Harris-Typus-II-Frakturen diagnostiziert (Tab. 2).

Salter-Harris-Typus-III-Frakturen werden relativ selten angetroffen (Watkins und Auer, 1984; Embertson et al., 1986 a, b). Diese Fraktur beginnt im Gelenk und bricht durch den Gelenksknorpel, die Epiphyse und entlang der Zellmaturationszone in der Epiphysenfuge, wo sie wieder die Oberfläche des Knochens erreicht (Abb. 4). Scher- oder Torsionskräfte, welche in einem Gelenk entstehen, sind in den meisten Fällen für die Frakturen verantwortlich (Salter, 1980; Braden, 1981). Die Proznose dieser Frakturen muß vorsichtig gestellt werden, da das Gelenk betroffen ist und bei der Behandlung von solchen Frakturen eine perfekte Reduktion der Fraktur erfolgen muß, um die Entwicklung von degenerativen Gelenkserkrankungen zu verhindern. Dazu kommt, daß die tieferen Schichten der Wachstumsfuge wie auch die Blutzufuhr zur Epiphyse und Epiphysenfuge durch die Frakturlinie beeinträchtigt werden. Dies kann zu einem lokalen Epiphysenschluß und einer anschließenden Wachstumsstörung beitragen (Salter, 1980).

In einem Zeitraum von 10 Jahren wurden an der TexasA\&M-Universitätsklinik $14 \%$ Salter-Harris-Typus-IIIFrakturen diagnostiziert. Embertson et al. (1986 a) berichteten im gleichen Zeitraum über $11 \%$ (Tab. 2). Beim Menschen wird die Häufigkeit mit $8 \%$ (Rogers, 1970) und beim Hund mit $2 \%$ (Marretta und Schrader, 1983) angegeben (Tab. 2).

Salter-Harris-Typus-IV-Frakturen haben wie die Typus-IIIFrakturen ein intraartikuläres Fragment. Die Fraktur beginnt im Gelenk, durchbricht die Epiphyse, die Knorpelfuge und die Metaphyse, wo sie aus dem Knochen ausbricht (Abb. 5). Ähnliche Kräfte wie bei den Salter-Harris-TypusIII-Frakturen sind für die Entstehung von Salter-Harris-Typus-IV-Frakturen verantwortlich. Die Salter-Harris-TypusIV-Frakturen entstehen häufiger bei älteren Fohlen, bei welchen die Widerstandsfähigkeit der Epiphysenfuge sich derjenigen des umliegenden Knochens nähert. Auch bei diesen Frakturen muß eine vorsichtige Prognose für komplikationslose Heilung aus den gleichen Gründen wie bei Salter-Harris-Typus-III-Frakturen gestellt werden (Salter, 1980; Weber, 1980; Tachidjian, 1972).

In Tabelle 2 wird für den Menschen eine Häufigkeit des Auftretens einer Salter-Harris-Typus-IV-Epiphysenfraktur mit $10 \%$ (Rogers, 1970), beim Hund mit $24 \%$ (Marretta und Schrader, 1983) und beim Pferd mit $11 \%$ (Embertson et al., 1986 a) angegeben (Tab. 2). Wir haben in $7 \%$ aller Epiphysenfrakturen eine Typus-IV-Fraktur diagnostiziert (Tab. 2).

Salter-Harris-Typus-V-Frakturen können nicht als eigentliche Frakturen angesehen werden. Es tritt vielmehr eine lokale Zerstörung der Wachstumsplatte durch starke plötzliche Druckbelastung auf (Abb. 6). Die Folge dieses akuten Traumas ist eine Zerstörung der Blutgefäße der Epiphysenfuge, welches eine einseitige Verknöcherung der Epiphysenfuge und die Entwicklung von angulären Achsenfehlstellungen einleitet (Salter, 1980; Tachidjian, 1972). SalterHarris-Typus-V-Frakturen treten beim Junghund nicht sel-

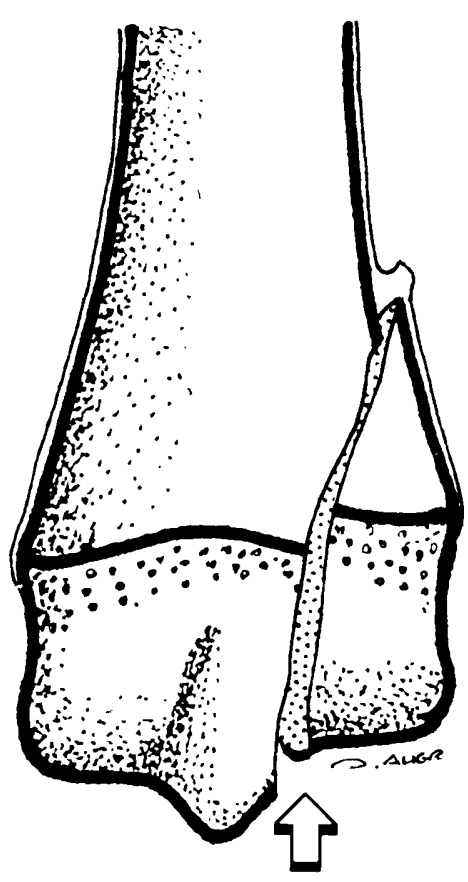

Abb. 5: Skizze einer Salter-Harris-Typus-IV-Epiphysenfraktur. Das aus epiphysealen und metaphysealen Anteilen bestehende Fragment, dessen Fraktur durch eine Kraft vom Gelenk aus verursacht wurde (Pfeil), ist nach proximal verschoben. Dies verursachte eine Asymmetrie des Gelenks.

ten an der distalen Epiphyse der Ulna auf (Newton et al., 1975).

Die Prognose von Salter-Typus-V-Frakturen ist gravierend. Frakturen dieser Art müssen auf jeden Fall, wenn eine komplikationslose Heilung angestrebt wird, chirurgisch behandelt werden (Auer und Martens, 1980; Fretz und Mcll. wraith, 1982).

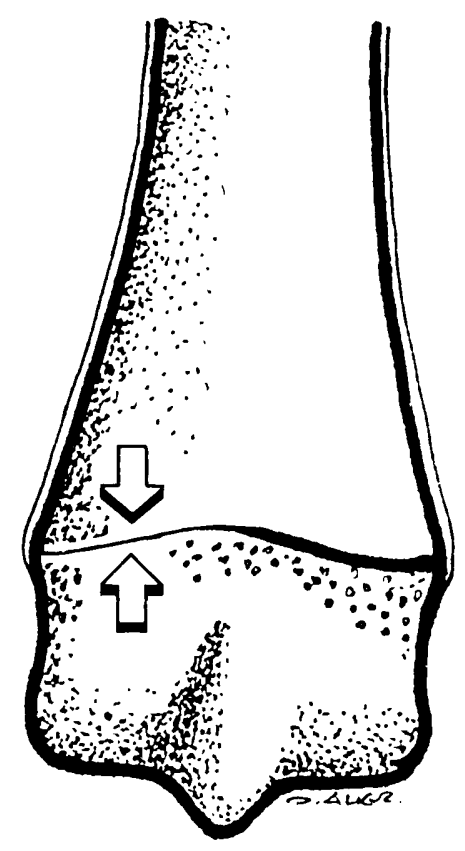

Abb. 6: Skizze einer Salter-Harris-Typus-V-Epiphysenfraktur. Lokale Krafteinwirkung (Pfeile) zerstörte die Epiphysenfuge und verursachte einen vorzeitigen Schluß der Epiphysenfuge. 
In Tabelle 2 werden die Salter-Harris-Typus-V-Frakturen nicht aufgeführt, da diese Diagnose erst zurückblickend gestellt werden kann, wenn eine Achsenfehlstellung schon vorhanden ist. Doch werden in Tabelle 2 einige Frakturen aufgeführt, welche nicht einem Typus zugeordnet werden konnten, da sie aus einer Kombination von Salter-HarrisFrakturtypen bestanden.

In der Literatur wird auch eine Salter-Harris-Typus-VIFraktur beschrieben (Salter und Harris, 1963; Llewellyn, 1976; Fackelman, 1984), doch wird diese Art von Epiphysenfraktur als Teil der Salter-Harris-Typus-V-Fraktur angesehen (Salter, 1980).

\section{Behandlung}

Die Frakturheilung beim wachsenden Individuum geht schneller vor sich als beim ausgewachsenen Menschen oder Tier (Salter, 1980; Tachidjian, 1972). Dies ist darauf zurückzuführen, daß das Periost und Endost des wachsenden Individuums eine höhere osteogene Aktivität aufweist als das des ausgewachsenen Menschen oder Tieres (Salter, 1980). Die Salter-Harris-Typus-I-Frakturen können theoretisch am schnellsten heilen, da dauernd neue Zellen in der Epiphysenfuge gebildet werden, die dann in den Zonen der primären Knochenformation abgebaut und in Knochen umgewandelt werden. Deswegen wird die Frakturstelle relativ schnell in Knochen umgewandelt, sofern die Fraktur stabilisiert werden kann. Salter (1980) beschrieb, daß beim Menschen Salter-Harris-Typus-I-, -II- oder -III-Epiphysenfrakturen innerhalb von 3 Wochen heilen können. Dies dauert beim Pferd jedoch etwas länger (Watkins et al., 1985; Embertson et al., 1986 b). Nach erfolgreicher Reduktion von Salter-Harris-Typus-I-, -II- oder -III-Frakturen kann auch beim Fohlen eine schnelle Heilung erfolgen (Embertson et al., 1986 b).

Um jedoch eine optimale Frakturheilung zu erreichen, müssen die Grundsatzprinzipien der Frakturbehandlung eingehalten werden (Müller et al., 1979; Turner et al., 1976; Turner, 1982 a; Fackelman und Nunamaker, 1982). Diese Prinzipien bestehen aus anatomischer Reduktion der Frakturfragmente und solider Stabilisation der Fraktur. Alle Reduktionen, ob sie konservativ durch Zug oder durch chirurgischen Eingriff erreicht werden, sollten so atraumatisch wie möglich durchgeführt werden (Tachidjian, 1972). Verletzung des delikaten Epiphysenknorpels oder der Blutversorgung der Epiphyse sollten unter allen Umständen vermieden werden. Deswegen sollten keine kraftvollen Manipulationen mittels chirurgischer Instrumente, welche direkten Druck auf die Epiphysenfuge ausüben, durchgeführt werden (Salter und Harris, 1963; Tachidjian, 1972). Frakturen in der Region der Epiphyse sollten so schnell wie möglich operiert und stabilisiert werden. Jedes Zuwarten des operativen Eingriffs bringt weitere Verletzungen der Epiphysenfuge mit sich, was eventuelle Wachstumsstörungen zur Folge haben könnte (Tacbidjian, 1972).

Stabilisation für den Transport in die Klinik ist ein Problem beim Großtier (Bramlage, 1983). Beim Hund und Menschen können Frakturen für den Transport gut stabilisiert und weitere Bewegungen in der Frakturebene zum größten Teil verhindert werden. Deswegen ist auch die Prognose beim Kleintier und beim Menschen besser zu stellen als beim Großtier (Watkins und Auer, 1984).

Für den Transport in eine Klinik sollten die Fohlen unter allen Umständen mit einer Schiene oder einem Fiberglasverband temporär fixiert werden. Dies ist vor allem wichtig in den Fällen, in denen die distalen Anteile der GliedmaBen frakturiert sind. Schwierig wird eine solche Fixation bei Humerus-, Radius-, Femur- und Tibiafrakturen. In diesen Fällen sollte eine Schiene an der lateralen Seite der Gliedmaße angebracht werden und bis auf die Höhe der Schulter oder Hüfte hochgezogen werden. Dies erlaubt die Belastung der frakturierten Gliedmaße, was für einen Transport in eine Klinik notwendig ist (Bramlage, 1983).

Nach 10 Tagen ist es schwierig, eine Epiphysenfraktur zu reduzieren, ohne daß der Epiphysenfuge Schaden zugefügt wird; es werden doch zu diesem Zeitpunkt sehr große Kräfte benötigt, um eine anatomische Reduktion zu erreichen (Tachidjian, 1972). Zu diesem Zeitpunkt ist es in den meisten Fällen besser, auf eine anatomische Reduktion der Fraktur zu verzichten und eine eventuell sich entwickelnde Achsenabweichung in einem zweiten Schritt mittels einer korrektiven Osteotomie zu behandeln (Tachidjian, 1972; Llewellyn, 1976).

Salter-Harris-Typus-III- und -IV-Frakturen müssen unter allen Umständen anatomisch reduziert werden, damit die Entwicklung von degenerativen Gelenkserkrankungen verhindert werden kann (Tachidjian, 1972). Obwohl auch perfekte Reduktion bei Salter-Harris-Typus-I- und -II-Frakturen erwünscht ist, ist es hier jedoch nicht unbedingt erforderlich. Residuale Achsenfehlstellungen scheinen sich durch kontinuierliches Wachstum auf der konkaven Seite des Knochens nach der Heilung zu korrigieren. Sollte solch eine Korrektur nicht eintreten, so kann durch eine korrektive Osteotomie die normale Gliedmaßenachse wiederhergestellt werden (Pollen, 1979).

Nichtchirurgische Korrektur von Epiphysenfrakturen sind bevorzugt, falls eine adäquate Reduktion erreicht werden kann, welche dem Knochen die Möglichkeit gibt, in einer normalen Konfiguration zu heilen (Salter, 1980). Bei den meisten Epiphysenfrakturen der Fohlen ist eine konservative Korrektur jedoch nicht möglich, und die anatomische Reduktion muß chirurgisch angestrebt werden (Watkins und Auer, 1984).

Epiphysenfrakturen des proximalen Radius, des Humerus, Femurs und der proximalen Tibia sollten nicht durch einen Fiberglasverband behandelt werden, da diese Verbände an der Stelle aufhören, wo die Fraktur lokalisiert ist. Die Frakturstelle wird durch solche Verbände nicht fixiert; im Gegenteil, das zusätzliche Gewicht führt zu einer größeren Verschiebung der Frakturelemente und zu gravierenderer Traumatisierung (Bramlage, 1983).

Ein weiteres Problem ist die Fixation von Epiphysenfrakturen, da es in vielen Fällen nicht möglich ist, mit einem externen Fiberglasverband die Frakturelemente in einer reduzierten Konfiguration zu stabilisieren. Die chirurgische Versorgung von Epiphysenfrakturen ist insofern problematisch, als eine Fixation durch die Epiphysenfuge wenn immer möglich vermieden werden sollte (Campbell et al., 
1959). Falls dieses Ziel nicht erreicht werden kann, sollten die Implantate keine Schraubengewinde aufweisen und einen maximalen Durchmesser von $4 \mathrm{~mm}$ aufweisen (Campbell et al., 1959). Die Implantate sollten wenn möglich im rechten Winkel zur Epiphysenfuge eingesetzt werden (Campbell et al., 1959). Solche Implantate verhindern das Wachstum nur geringgradig und induzieren in den meisten Fällen keine Wachstumsstörungen. Diese theoretischen Prinzipien können jedoch in der Großtierpraxis in vielen Fällen nicht angewendet werden, da es vor allem beim Fohlen wichtig ist, wo immer möglich Knochen für die Fixation einer Epiphysenfraktur zu verwenden (Watkins und Auer, 1984; Watkins et al., 1985; Embertson et al., 1986 b). Dies verschlechtert die Prognose für einen künftigen Einsatz des Fohlens in einer athletischen Disziplin (Watkins und Auer, 1984).

Offene Frakturen treten relativ häufig in der Metakarpaloder Metatarsalregion auf (Turner, 1984; Bramlage, 1983). Dies geschieht hauptsächlich dadurch, daß in diesem Gebiet nur einige Sehnen, Bindegewebe und Haut den Knochen bedecken. Zugespitzte Knochensplitter können die Haut von innen heraus durchdringen und öffnen. Dasselbe Resultat kann durch scharfe Objekte von außen her erreicht werden. Eine offene Fraktur muß immer als kontaminiert oder sogar infiziert angesehen und behandelt werden.

In der Folge werden die verschiedenen Frakturtypen in den Epiphysenregionen, die in den einzelnen Knochen auftreten können, diskutiert und Möglichkeiten zu deren Fixation kurz beschrieben.

\section{Skapula}

Die Skapula besitzt verschiedene Epiphysen, von welchen nur der Tuber scapulae für den Tierarzt wichtig erscheint. Diese Zugepiphyse besteht aus 2 separaten Ossifikationszentren, die schon mit 3 Monaten miteinander verwachsen (Schmidt, 1960; Eulner, 1974). Die am häufigsten angetroffene Epiphysenfraktur der Skapula besteht aus einer Abrißfraktur des Tuber scapulae, da dieses die Ansatzstelle der starken Sehne des M. biceps brachii ist. Salter-Harris-Typus-I- oder -II-Frakturen werden in dieser Region angetroffen. Solche Frakturen treten hauptsächlich bei Jungtieren zwischen dem ersten und zweiten Lebensjahr auf und verursachen eine gravierende Lahmheit.

Unter den 22 Skapulafrakturen, welche seit 1978 an der Texas-A\&M-Universitätsklinik diagnostiziert wurden, befanden sich 9 Epiphysenfrakturen (Tab. 1).

Die Reduktion der Fraktur und Fixation ist nur erfolgreich, wenn die Behandlung sofort eingeleitet wird und während des chirurgischen Eingriffs gleichzeitig auch die Sehne des M. biceps brachii durchtrennt wird (Leitch, 1977). Wird diese Sehne nicht durchtrennt, muß mit einem postoperativen Niederbruch der Fixation gerechnet werden, da die Implantate der enormen Zugskraft nicht widerstehen können. In der Heilungsphase wächst die Sehne wieder zusammen, doch ist sie nicht mehr so stark wie vorher. Resektion der ganzen Epiphyse ist eine weitere Behandlungsmöglichkeit, doch können die Tiere nicht als Gebrauchspferde eingesetzt werden (Wagner et al., 1985). Die konservative Behandlung ist in den meisten Fällen erfolglos, da eine Arthrose im Schultergelenk auftreten kann, die oft von Stelzfußbildung begleitet wird.

\section{Humerus}

Epiphysenfrakturen des Humerus treten sehr selten auf. Unter den 40 seit 1978 an der Texas-A\&M-Universitätsklinik diagnostizierten Humerusfrakturen befanden sich 6 Epiphysenfrakturen (Tab. 1). Davon wurden 4 Frakturen in der proximalen Epiphyse angetroffen, $1 \mathrm{im}$ Tuberculus majus und 1 am medialen Epicondylus des distalen Humerus.

Die klinischen und röntgenologischen Befunde im Zusammenhang mit Epiphysenfrakturen des proximalen Humerus bestehen aus minimaler Verschiebung der Epiphyse des Humeruskopfes oder proximaler Distraktion des Tuberculum majus (Embertson et al., 1986 a). Humeruskopffrakturen sollten konservativ mit Stallruhe und Tuberculum-majus-Frakturen mit Zuggurtung behandelt werden. Abrißfrakturen der Zugsepiphyse des medialen Epicondylus des distalen Humerus werden auch von Embertson et al. (1986 a, b) erwähnt. Diese Apophyse bildet die Ansatzstelle des tiefen und oberflächlichen Zehenbeugers sowie des M. flexor carpi radialis und M. flexor carpi ulnaris. Diese Muskeln erschweren eine anatomische Reduktion, und deswegen kann nicht mit einer guten Prognose gerechnet werden.

Epiphysenfrakturen der Trochlea humeri wurden von Embertson et al. (1986 a, b) erwähnt, doch gaben diese Autoren eine schlechte Prognose für Heilung solcher Frakturen mit oder ohne Osteosynthese. Salter-Harris-Frakturen des Typus I, II, und IV wurden an dieser Stelle diagnostiziert. Beide Salter-Harris-Typus-IV-Frakturen waren am lateralen Epicondylus lokalisiert.

\section{Radius}

Epiphysentrakturen können in der proximalen und distalen Epiphyse auftreten. In der proximalen Epiphysenregion wurden Salter-Harris-Frakturen des Typus I bis IV diagnostiziert (Embertson et al., 1986 a, b; Gaines und Auer, 1983). Die meisten Frakturen in dieser Gegend entstehen durch Unfälle und müssen mit Osteosynthese behandelt werden. Dabei stellt sich oft das Problem der Art der Fixation. Bei Salter-Harris-Typus-I- oder -II-Frakturen ist die Epiphyse oftmals verschoben. Mit Hilfe des Müller-Fragment-Distraktors oder der Becken-Reduktionszange kann solch eine Fraktur relativ leicht reduziert werden. Bei älteren Fohlen kann die Ulna zur Fixation herangezogen werden, indem eine Platte über deren kaudalem Rand angebracht und mittels Schrauben mit dem Radius verankert wird. Bei jungen Fohlen ( $<$ als 10 Monate alt) sollte die Ulna nicht mittels Schrauben an den Radius fixiert werden, da diese beiden Knochen verschiedene Wachstumsraten aufweisen. Sollte die Ulna zu diesem Zeitpunkt an den Radius fixiert werden, entsteht eine Subluxation und Arthrose im Kubitalgelenk (Stover und Rick, 1985).

Weitere Implantate wie T-Platten können die Epiphyse an den Radius fixieren. In Salter-Harris-Typus-II-Frakturen sollten wenn möglich Zugschrauben in den metaphysealen 


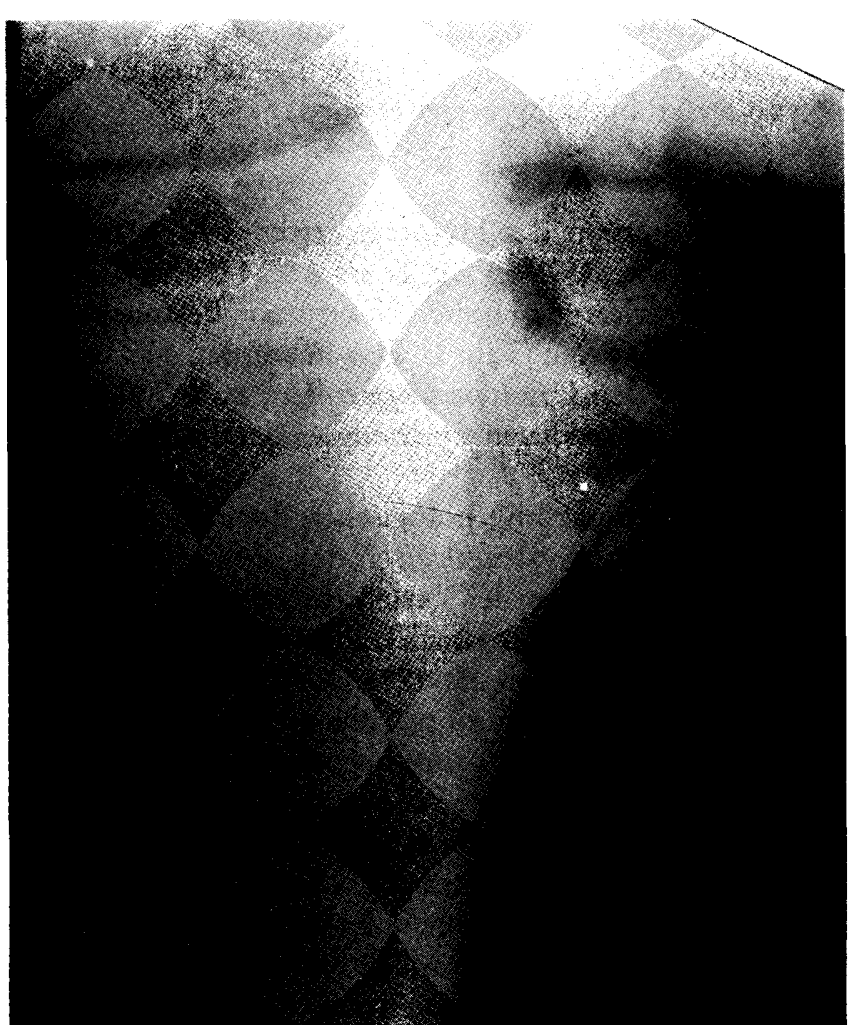

Abb. 7: Kraniokaudales Röntgenbild der proximalen Epiphysenregion des rechten Radius eines einjährigen Vollblutpferdes mit einer Salter-Harris-Typus-III-Epiphysenfraktur.

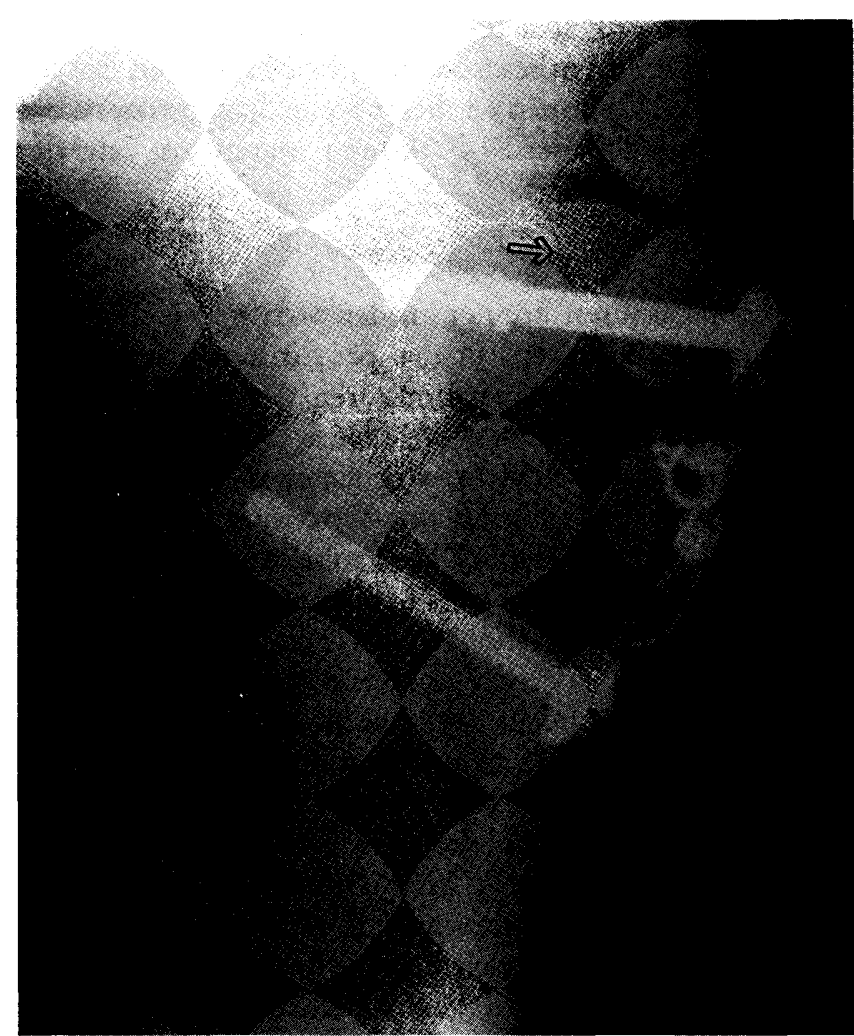

Abb. 8: Kraniokaudales Röntgenbild des gleichen Pferdes wie in Abb. 710 Monate nach Fixation der Fraktur. Eine Zugschraube mit einer Unterlagsscheibe wurde in die Epiphyse implantiert. Der einseitige Zug des lateralen Kollateralbandes wurde durch Zuggurtung neutralisiert. Die Frakturlinie ist beinahe verheilt (Pfeil). Das Pferd gewann 3 Monate später ein Rennen.

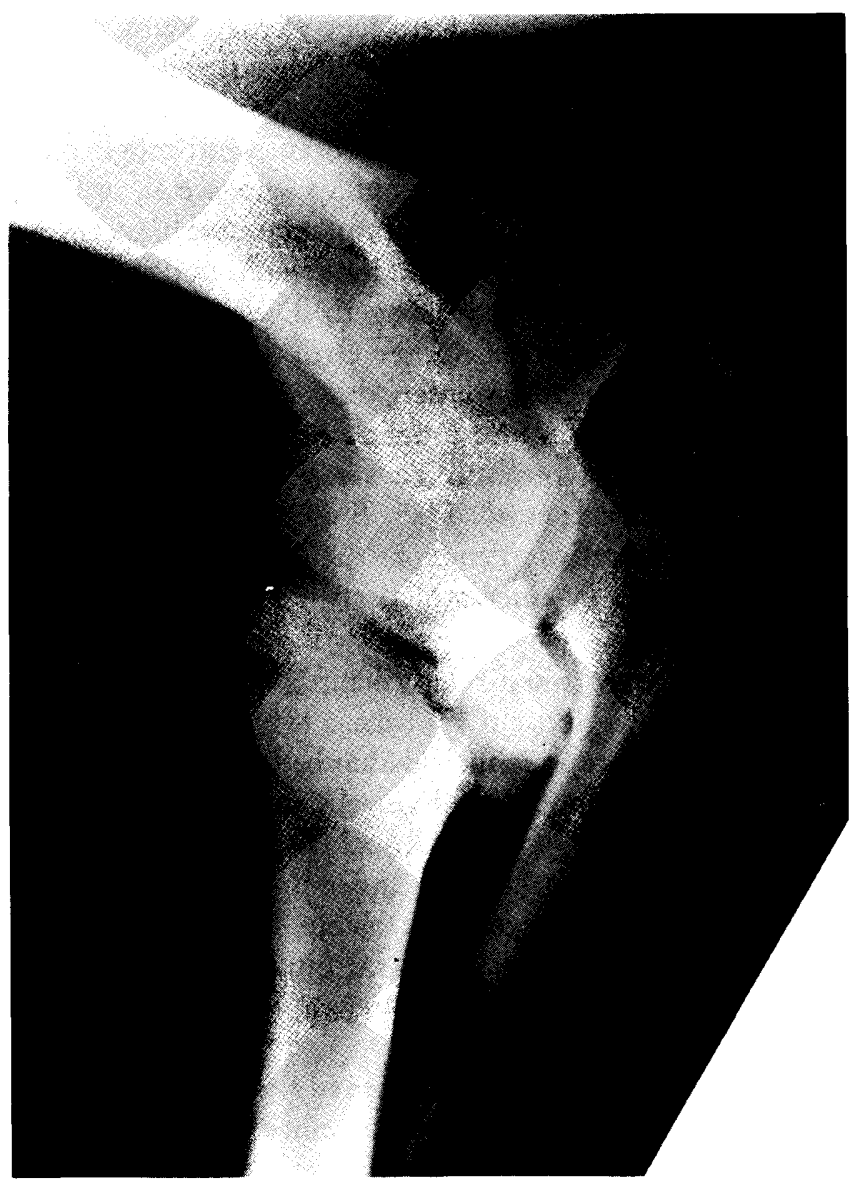

Abb. 9: Lm-Röntgenbild des linken Ellbogenbereiches eines 5 Monate alten Araberfohlens mit einer Salter-Harris-Typus-IV-Epiphysenfraktur. Die Ulna ist zusammen mit dem Fragment des proximalen Radius und dem Humerus nach kaudal verschoben.

Anteil des Epiphysenfragments implantiert werden. In diesen Fällen ist es möglich, daß keine T-Platten zwischen Epiphyse und Radius eingesetzt werden müssen, was die Prognose verbessert.

Bei Salter-Harris-Typus-III-Frakturen sollte das Zuggurtungsprinzip mittels Schrauben und Draht oder einer dünnen Platte eingesetzt werden (Abb. 7, 8) (Gaines und Auer, 1983). Durch Hebelwirkung sollte versucht werden, eine perfekte anatomische Reduktion zu erreichen, doch kann diese nicht mittels einer Arthrotomie bestätigt werden. Deswegen ist eine Arthrotomie in diesen Fällen nicht angezeigt.

Salter-Harris-Typus-IV-Frakturen werden in den meisten Fällen in der Frontalebene angetroffen (Abb. 9). Bei diesen Frakturen ist die Ulna zusammen mit dem Radiusfragment nach kaudal verschoben. Solche Frakturen sind schwierig zu behandeln, und absolute Kongruenz im Ellbogengelenk muß erreicht werden, um die Entwicklung einer Arthrose zu verhindern. Durch Zugschrauben kann das Fragment an den Radius fixiert werden, doch muß oft die Ulna mit in die Fixation einbezogen werden, was zu Komplikationen führen kann (Abb. 10).

Distale Epiphysenfrakturen im Radius werden relativ selten diagnostiziert und sollten mittels Osteosynthese behandelt werden. Von 8 Epiphysenfrakturen, die sich unter den an der Texas-A\&M-Universitätsklinik seit 1978 diagnosti- 


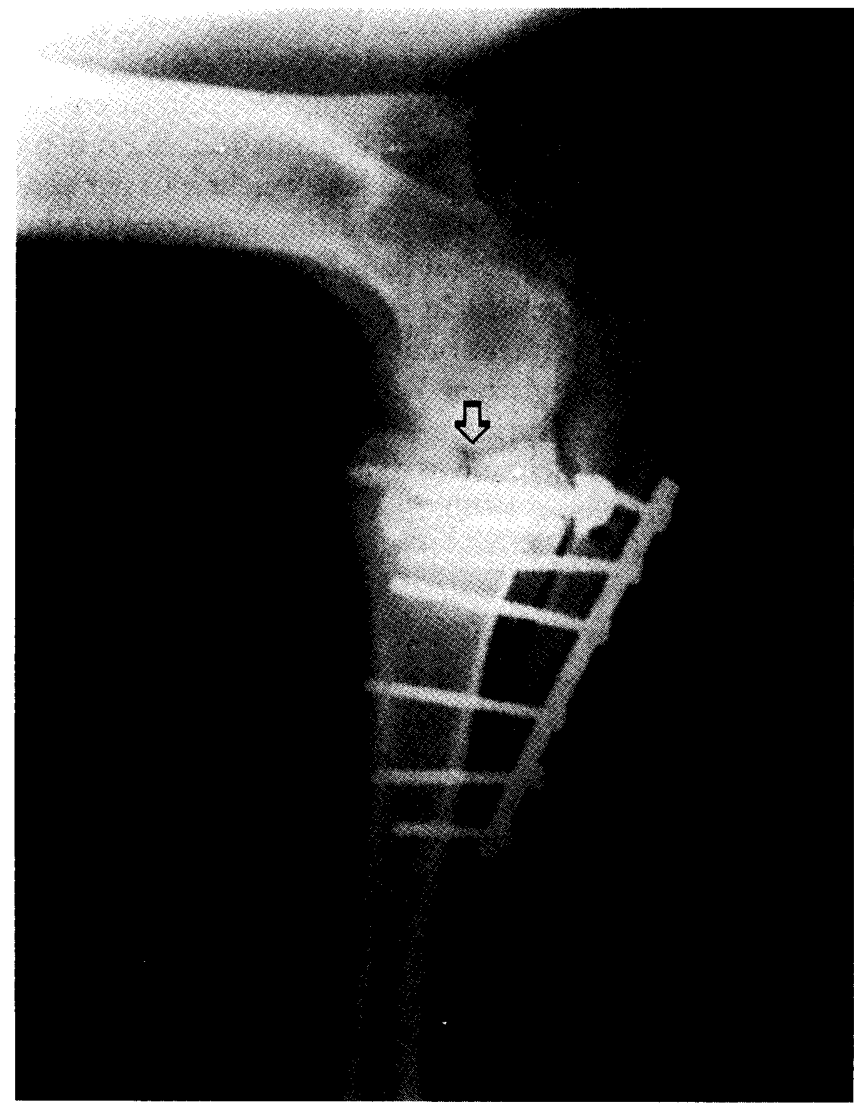

Abb. 10: Postoperatives Lm-Röntgenbild des gleichen Fohlens wie in Abb. 9. Durch Zugschrauben wurde versucht, das Radiusfragment an den Rest des Radius zu fixieren. Durch eine schmale DCP wurden von kaudal Zugschrauben durch die UIna in den Radius implantiert. Ungenügende Reduktion der Fraktur und Fixation in dieser Konfiguration verursachten eine Gelenkasymmetrie (Pfeil) und eine Arthrose.

zierten 35 Radiusfrakturen befanden, war nur 1 in der distalen Epiphyse lokalisiert (Tab. 1).

Salter-Harris-Typus-I- (Abb. 11) und -II-Frakturen treten bei jüngeren, während -Typus-III- und -IV-Frakturen bei älteren Fohlen auftreten.

Bei jüngeren Fohlen kann versucht werden, diese Frakturen konservativ zu behandeln durch Anbringen eines Fiberglasverbandes, doch treten dabei oftmals Komplikationen auf.

Bei der Osteosynthese dieser Frakturen muß in den meisten Fällen die Epiphysenfuge temporär durch eine Platte oder andere Implantate überbrückt werden (Abb. 12). Diese wachstumshemmenden Implantate sollten jedoch so früh wie möglich entfernt werden, um die Entwicklung einer Achselfehlstellung zu vermeiden (Abb. 13).

\section{Ulna}

Bei Frakturen der Ulna kann die Salter-Harris-Klassifikation nicht immer angewendet werden. Bei dieser Epiphyse

Abb.12: Dp-Röntgenbild des gleichen Fohlens wie in Abb. 11 nach erfolgter Fixation. Mittels einer T-Platte an der medialen Seite wurde die Fraktur fixiert. 2 Spongiosaschrauben wurden durch die Platte in die Epiphyse und 3 Kortikalisschrauben in die Metaphyse implantiert. Dies erlaubte normale Belastung der Gliedmaße. Die distale Schraube in der Metaphyse wurde eine Woche nach der Operation durch eine kurze Spongiosaschraube ersetzt, um eine Wachstumshemmung auf der lateralen Seite zu verhindern.

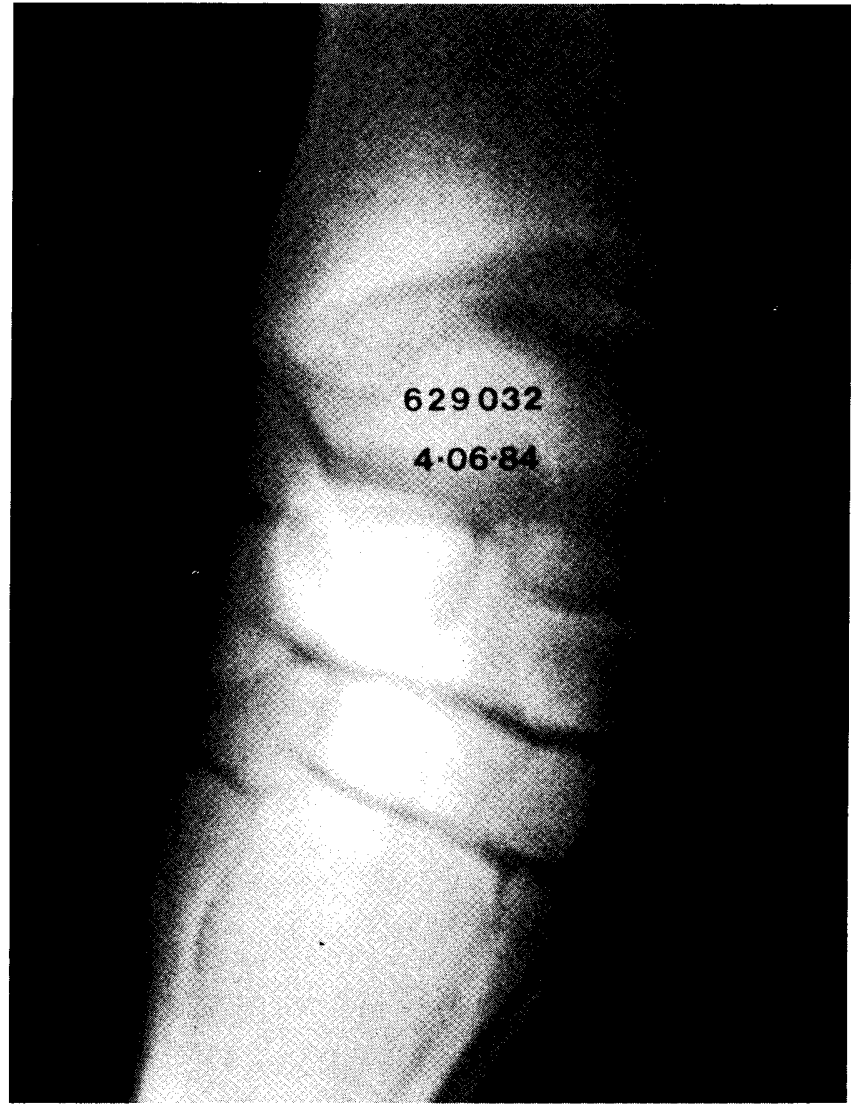

Abb. 11: Dp-Röntgenbild der rechten Karpalregion eines 2 Wochen alten Quarter-Horse-Fohlens mit einer Salter-Harris-Typus-I-Epiphysenfraktur des distalen Radius.

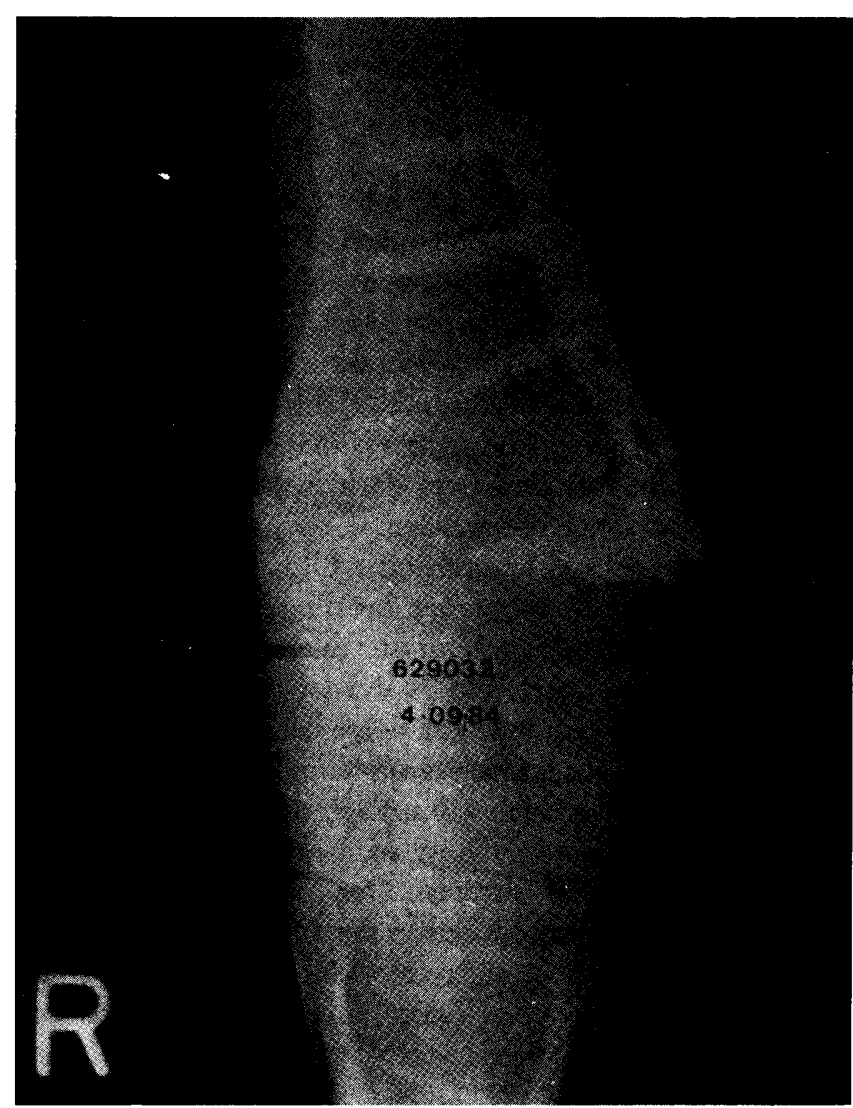




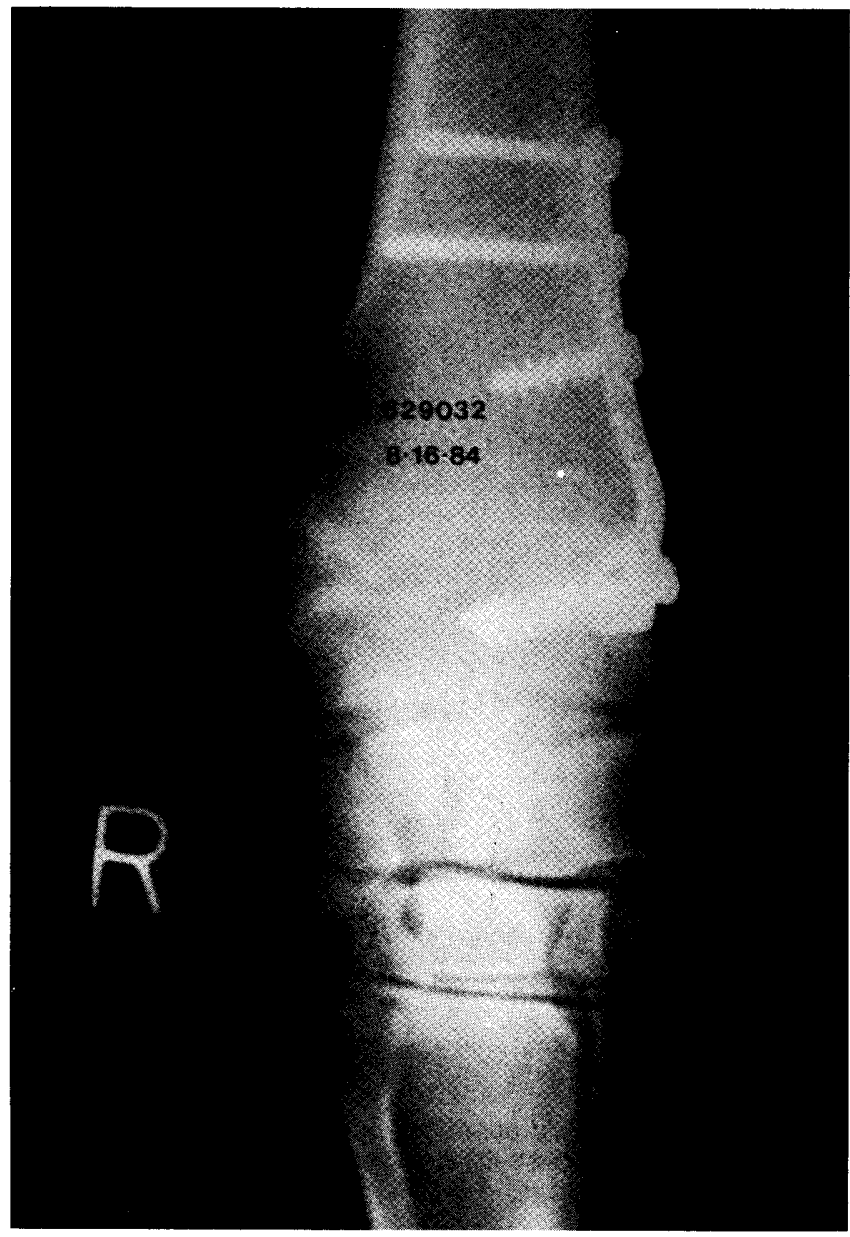

Abb. 13: Dp-Röntgenbild des gleichen Fohlens wie in Abb. 11 und 124 Monate später. Da die Implantate nicht wie instruiert nach 2 Monaten entfernt wurden, entwickelte sich eine Varus-Achsenfehistellung. Man beachte, daß die Wachstumsplatte auf der medialen Seite obliteriert ist.

handelt es sich um eine Zugepiphyse, die nicht mit einem Gelenk verbunden ist. Salter-Harris-Typus-I- und die meisten -Typus-II-Frakturen können anhand der klassischen Definition beschrieben werden (Abb. 14). Doch treten spezifische Salter-Harris-Typus-II-Frakturen auf, bei denen der Metaphysenanteil bis ins Kubitalgelenk reicht. Da nach der Definition von Salter und Harris (1963) eine Typus-II-Fraktur kein artikuläres Fragment enthält, können diese Frakturen nicht so eingeteilt werden.

Fohlen mit einer Olekranon- oder Ulnafraktur haben eine charakteristische Stellung der beteiligten Gliedmaße. Da der Zug des M. triceps brachii nur die Epiphyse verschiebt, kann der Karpus nicht fixiert werden, was eine Nichtbelastbarkeit der Gliedmaße zur Folge hat. Durch Anbringen einer Schiene in der Karpalregion kann dieser Zustand temporär korrigiert werden (Bramlage, 1983). Die Verschienung der Vordergliedmaße ist auch eine mögliche Behandlungsart für solche Frakturen. Der Nachteil davon ist die länger dauernde Immobilisation der fixierten Gelenke, was Degenerationserscheinungen im Gelenkknorpel und in den periartikulären Geweben hervorrufen kann. Im weiteren können Drucknekrosen der Haut auftreten (Bramlage, 1983).
Die Epiphyse bei jungen Fohlen bis zu einem Alter von 5 Wochen besitzt um den zentralen Ossifikationskern einen relativ breiten Knorpelring. Epiphysenfrakturen bei solchen Fohlen sind schwierig zu behandeln. Das Problem besteht darin, daß die Knorpelschicht weicher als der zentrale Knochenkern ist. Wird nun ein Implantat angebracht, so kann dieses nicht direkt auf dem Knochenkern befestigt werden, da die Knorpelschicht dazwischen liegt, was einen postoperativen Zusammenbruch der Fixation zur Folge haben kann. Zur chirurgischen Versorgung dieser Frakturen sollte das Zuggurtungsprinzip angewendet werden. $\mathrm{Zu}$ diesem Z weck können Schrauben und Draht oder Platten eingesetzt werden (Monin, 1978). Eine andere Möglichkeit bietet die Sulzer-Hacken-Platte, die aber vom Autor nicht erfolgreich angewendet werden konnte (Donecker et al., 1984). Postoperativ sollten, nach Ansicht des Autors, solche Frakturen für einige Zeit mit einer kaudal angebrachten Schiene versehen werden, damit plötzliche starke Zugkräfte auf die fixierte Olekranonfraktur etwas gemildert werden können.

Bei älteren Fohlen können Epiphysenfrakturen des Olekranon mit Platten im Zusammenhang mit Zugschrauben (Abb. 15, 16) (Colahan und Meagher, 1979; Donecker et al., 1984) versehen werden. Um eine maximale Kompression der Frakturstelle zu erreichen, kann neben der dynamischen Kompressionsplatte auch noch ein zusätzliches Kompressionsinstrument angewendet werden.

Auch bei diesen Frakturen ist es wichtig, daß wenn immer möglich die Schrauben nur in die Ulna implantiert werden.

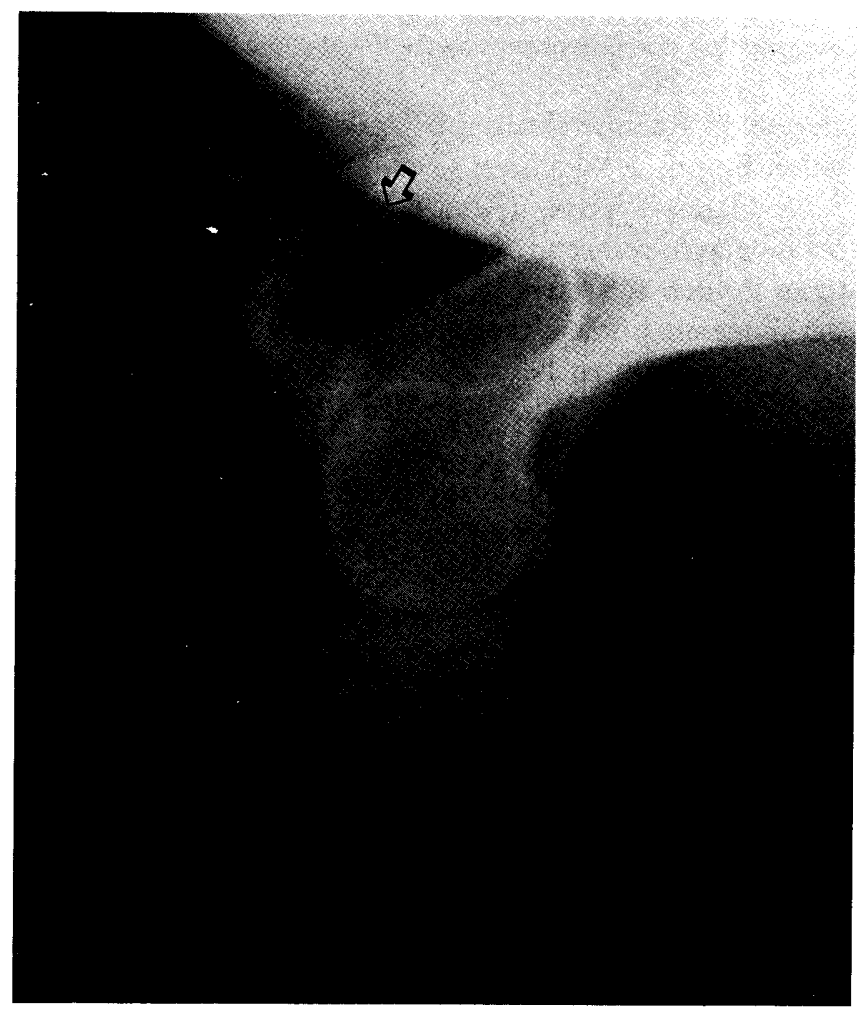

Abb. 14: Lm-Röntgenbild des Ellbogenbereiches eines 18 Monate alten Vollblutpferdes mit einer Salter-Harris-Typus-II-Epiphysenfraktur des Tuber olecrani. Das Fragment ist nach kranial verschoben. Man beachte das kleine Knochenfragment, das weiter nach kranioproximal verschoben ist (Pfeil). 
Eine eventuelle Transfixation von Ulna und Radius kann, wie bereits erwähnt, Komplikationen mit sich bringen (Stover und Rick, 1985). Seit 1978 wurden 61 Ulna- oder Olekranonfrakturen an der Texas-A\&M-Universitätsklinik diagnostiziert; davon waren 22 Epiphysenfrakturen (Tab. 1). Daß mehr als ein Drittel der Ulnafrakturen in der Epiphyse lokalisiert waren, zeugt von der Häufigkeit dieser Art Fraktur.

\section{Metakarpus und Metatarsus}

Bei Geburt des Fohlens ist die proximale Epiphyse des Metakarpus und Metatarsus bereits mit der Metaphyse verwachsen. Deshalb treten Epiphysenfrakturen nur in der distalen Epiphyse dieser Knochen auf. Salter-Harris-TypusII-Frakturen werden am häufigsten angetroffen. Solche Frakturen können durch 2 Zugschrauben im metaphysealen Anteil der Fraktur des distalen Fragments fixiert werden. In solchen Fällen sollten Fiberglasverbände angebracht werden, um weiteren Halt für die Frakturheilung zu geben. Je nach Alter des Fohlens müssen diese Fiberglasverbände binnen 2 bis 4 Wochen gewechselt werden. Wird eine Platte angebracht, so sollten die Schrauben, welche eventuell in die Epiphyse implantiert werden, die Epiphyse ganz durchqueren, damit das Wachstum nicht einseitig verringert wird. Die Schrauben sollten aber so früh wie möglich wieder von der Epiphyse entfernt werden.

Eine Epiphysenfraktur des Metakarpus und 2 Epiphysenfrakturen des Metatarsus wurden seit 1978 an der TexasA\&M-Universitätsklinik diagnostiziert (Tab. 1). Während dieser Zeit wurden 65 Patienten mit Metakarpal- und Metatarsalfrakturen eingeliefert.

\section{Proximale Phalanx}

Frakturen der proximalen Epiphyse des Fesselbeins werden in den Vorder- und Hintergliedmaßen selten diagnostiziert (Embertson et al., 1986 a). Unter 38 Fesselbeinfrakturen befanden sich 3 Salter-Harris-Typus-III-Epiphysenfrakturen (Tab. 1). Chirurgische Versorgung verbessert die meistens schlechte Prognose nicht (Embërtson et al., 1986 b). Fohlen mit dieser Art Fraktur werden häufig vorerst konservativ behandelt, da in der Regel nicht sofort Röntgenaufnahmen gemacht werden. Erst später, wenn keine Besserung eintritt, wird eine röntgenologische Diagnose gestellt; doch ist es zu diesem Zeitpunkt zu spät, da der Gelenkknorpel schon teilweise zerstört ist.

Fiberglasverbände können angebracht werden, doch muß auch dabei eine zumindest vorsichtige Prognose für einen späteren Einsatz als Gebrauchspferd gestellt werden.

\section{Femur}

In unserer Verteilung der Epiphysenfrakturen war der Femur in den meisten Fällen betroffen, wurden doch 29 Epiphysenfrakturen unter 59 Femurfrakturen diagnostiziert (Tab. 1). Davon traten 15 Frakturen in der proximalen und 14 in der distalen Epiphyse auf.

Frakturen der proximalen Femurepiphyse entstehen meistens bei jungen Fohlen, die sich aufbäumen oder sich rückwärts überschlagen (Embertson et al., 1986 a). Konservative Behandlung mittels Stallruhe ist nicht erfolgreich. Deshalb

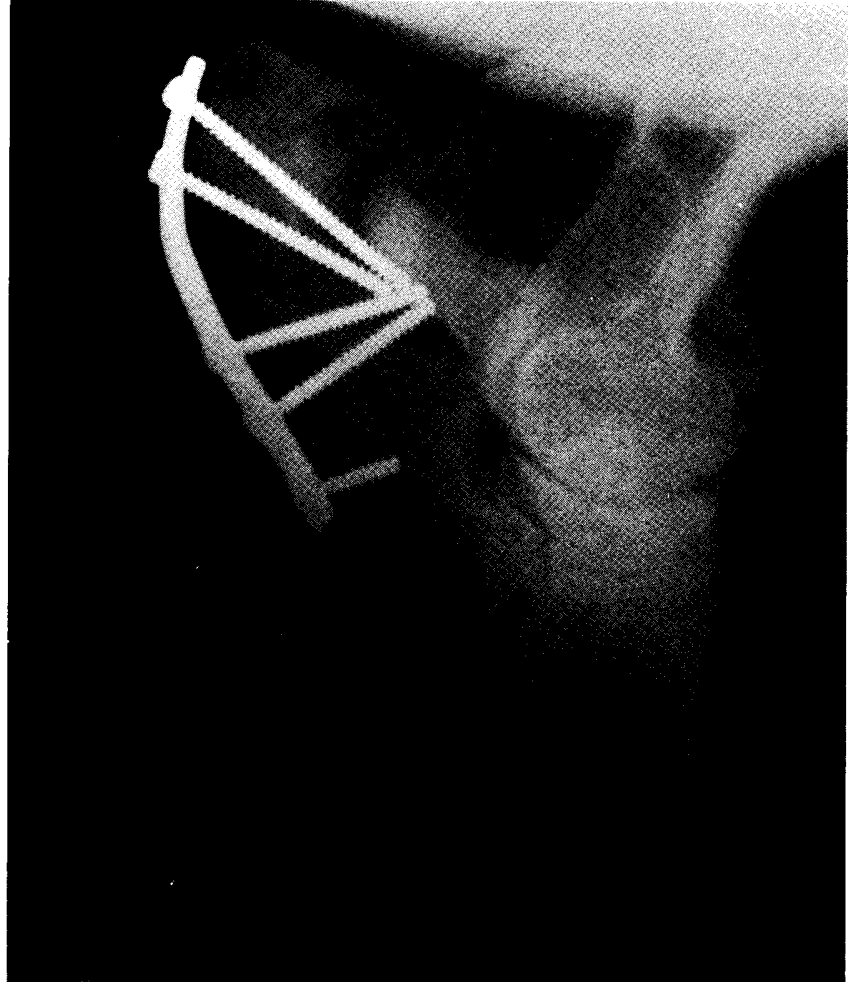

Abb. 15: Lm-Röntgenbild des gleichen Pferdes wie in Abb. 14 nach der Fixation der Fraktur. Eine schmale 5-Loch-DCP wurde von kaudal an der Tuber olecrani befestigt. Mittels 2 Zugschrauben wurde das Fragment an die Ulna fixiert. 4 Schrauben laufen zu einem gemeinsamen Punkt zusammen, was vermieden werden solite. Man beachte das kleine Knochenfragment, das nun wieder in der Region der Frakturlinie liegt (Pfeil). Da das Fragment nicht in unmittelbarer Nähe des Gelenks lokalisiert war, wurde es nicht entfernt.

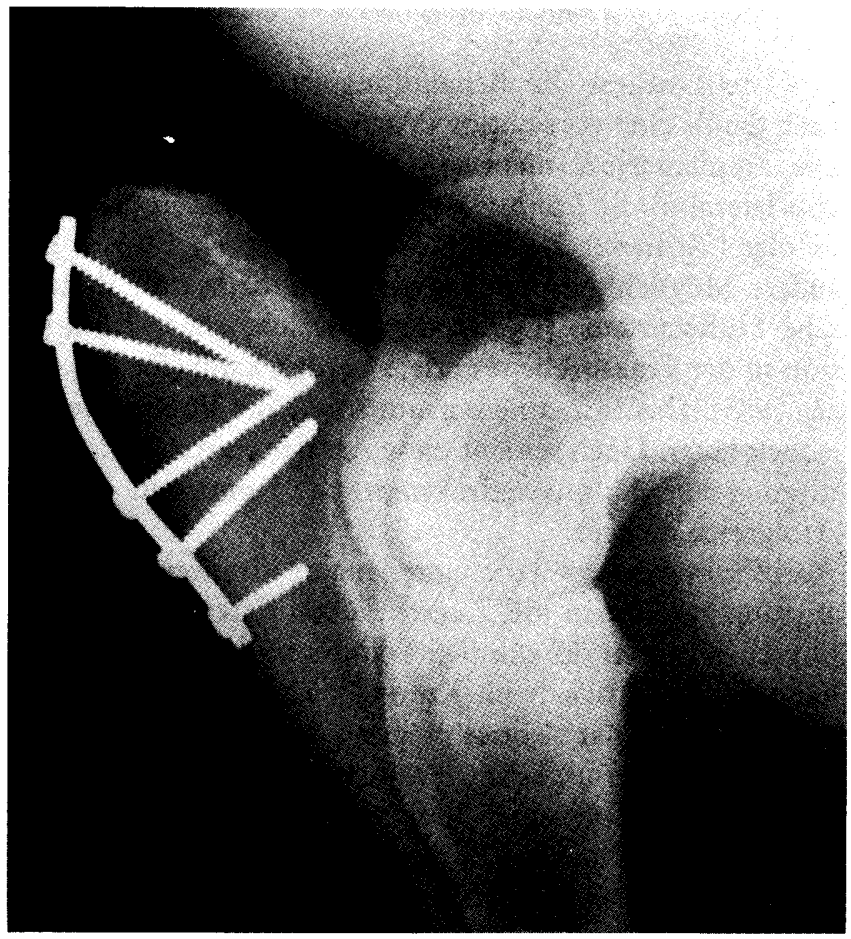

Abb. 16: Lm-Röntgenbild des gleichen Pferdes wie in Abb. 14 und 158 Monate nach Fixation der Fraktur. Die Fraktur ist verheilt und das kleine Knochenfragment mit dem Rest der Ulna verwachsen. Das Pferd wurde 4 Monate später erfolgreich in Rennen eingesetzt. 


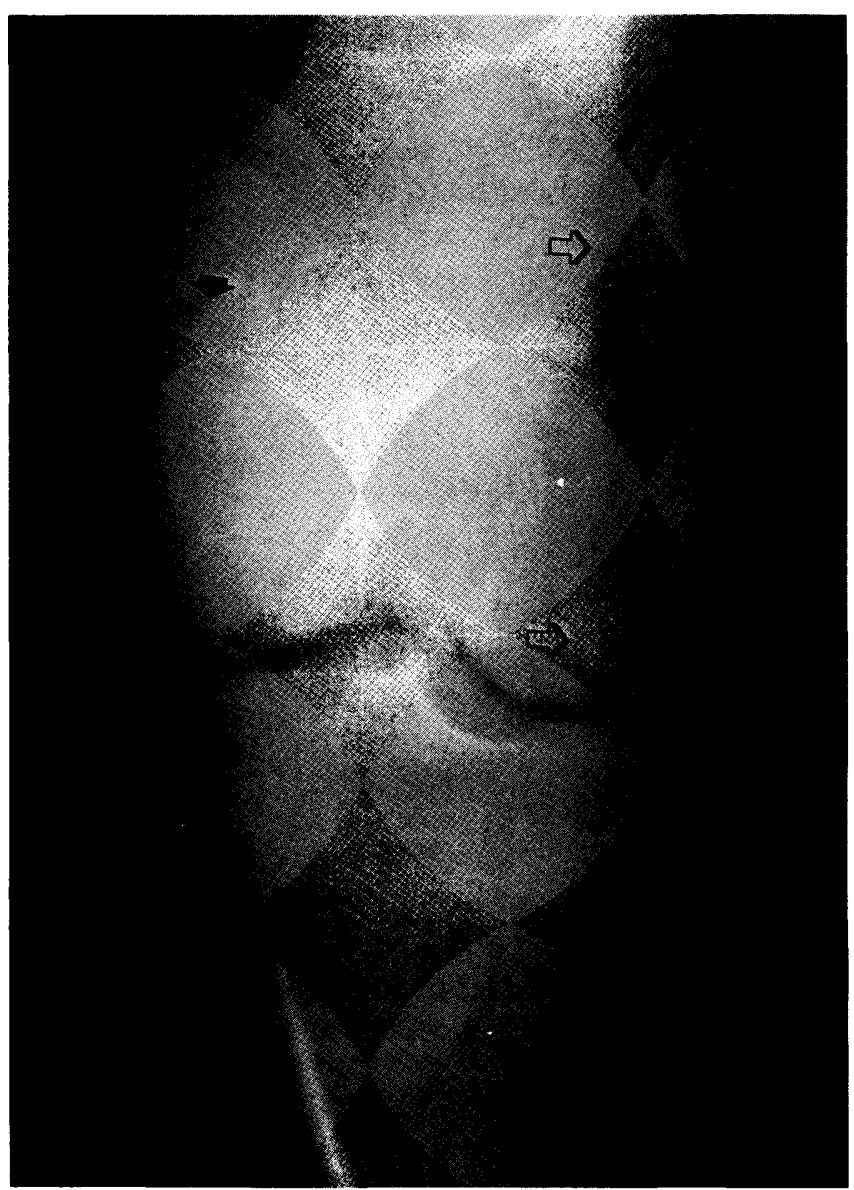

Abb. 17: Kaudokraniales Röntgenbild des distalen Femurs eines 5 Monate alten Araberfohlens mit einer Salter-Harris-Typus-IV-Epiphysenfraktur (offene Pfeile). In der gleichen Epiphyse wurde zusätzlich noch eine Salter-Harris-Typus-III-Fraktur diagnostiziert. Die Frakturlinie brach kranial in das Femoropatellargelenk ein (ausgefüllter Pfeil).

ist Osteosynthese die Behandlungsart der Wahl, obwohl auch damit eine vorsichtige Prognose gestellt werden muß. Die Implantation mehrerer paralleler Steinmann-Nägel von lateral in die Epiphyse verzeichnet zur Zeit die besten Erfolge (Turner et al., 1979; Embertson et al., 1986b). Eine andere Möglichkeit der Osteosynthese bringt die dynamische Hüftschraube (DHS), durch welche sogar Kompression in der Frakturebene erzielt werden kann (Regazzoni et al., 1985). Das Instrumentarium sowie die Implantate sind jedoch teuer. Doch scheint diese Art der chirurgischen Versorgung von Epiphysenfrakturen des Femurs in Zukunft erfolgreich zu sein.

Abrißfrakturen des Trochanter major können durch Zuggurtung zusammen mit Zugschrauben fixiert werden. Epiphysenfrakturen des distalen Femurs sind schwierig zu behandeln, da es sich beim Kniegelenk um ein Spiralgelenk mit exzentrischer Rotationsachse handelt (Abb. 17, 18). Dabei entstehen im kranialen Bereich der Epiphyse starke Zugkräfte, die bei einer Fraktur durch Implantate überbrückt werden müssen. Osteosynthesetechniken verschiedener Art wurden bei solchen Frakturen mit wenig Erfolg angewendet (Turner, 1982 c, 1984; Embertson, 1986 b). Die Kombination einer dynamischen Kondylenschraube (DCS) von lateral in den kranialen Teil der Epiphyse und einer DHS von kranial in den medialen Kondylus des Fe-

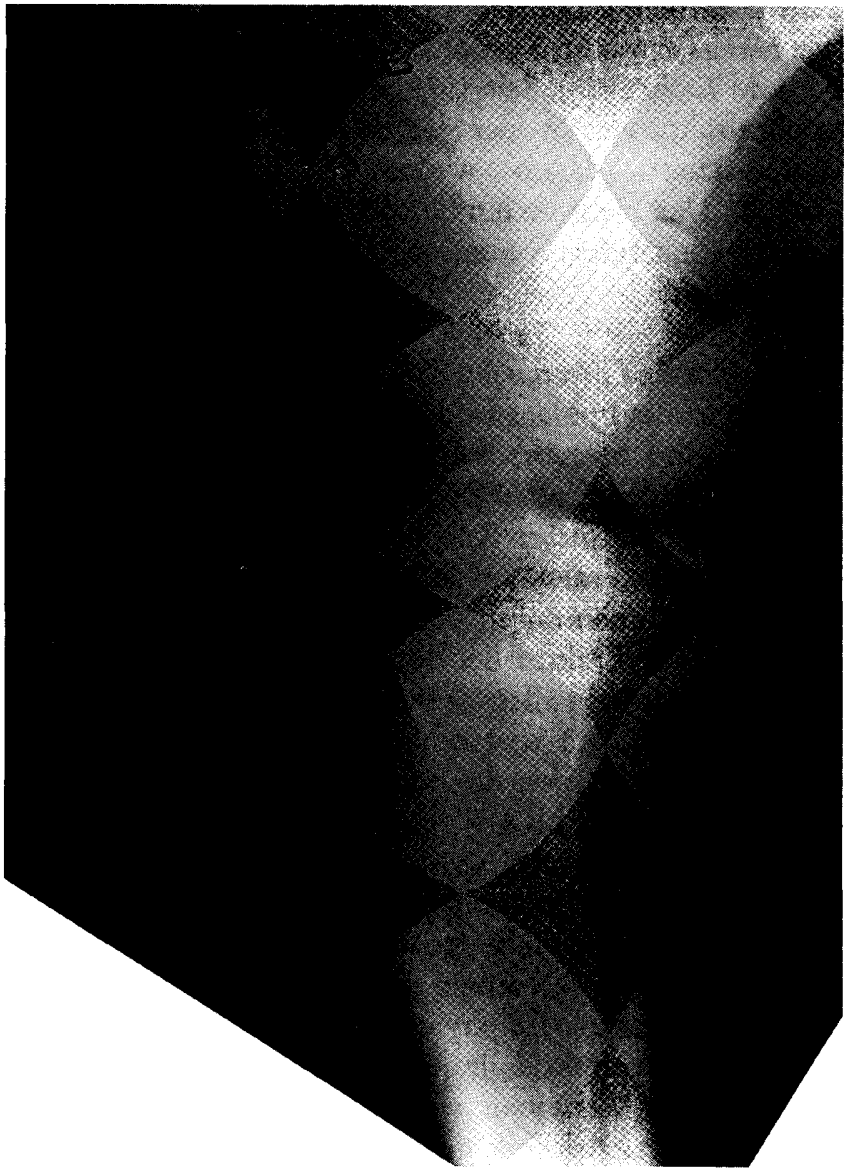

Abb. 18: Lm-Röntgenbild der gleichen Gelenkregion wie in Abb. 17. Man beachte die Frakturlinie im kranialen Anteil der Epiphyse (Pfeile) Die Fraktur wurde mittels Zuggurtung kranial und einer Platte medial fixiert. Das Pferd kann zur Zucht verwendet werden, zeigt aber eine Lahmheit.

murs scheint nach unseren ersten Versuchen eine stabile Lösung darzustellen.

\section{Tibia}

Frakturen der proximalen Epiphyse der Tibia sind häufig diagnostizierte Epiphysenfrakturen beim Fohlen (Watkins et al., 1985; Embertson et al., 1986a). Unter 55 Tibiafrakturen befanden sich 16 Epiphysenfrakturen, von welchen 11 in der proximalen Epiphyse, 1 in der Crista tibialis und 4 in der distalen Epiphyse lokalisiert waren (Tab. 1). Diese Frakturen, von denen die meisten vom Salter-Harris-Typus-II sind, können nicht konservativ durch Stallruhe behandelt werden (Abb. 19). Deshalb wurden verschiedene chirurgische Behandlungsmethoden entwickelt. Bei Fohlen, die jünger als einen Monat sind, können sich kreuzende Steinmann-Nägel von medial und lateral durch die proximale Epiphyse in die Metaphyse implantiert werden (Watkins et al., 1985). In vielen Fällen ist eine vollständige Reduktion nicht möglich, doch korrigieren sich die anfänglichen Achselfehlstellungen spontan durch verstärktes Wachstum auf der lateralen Seite. Fohlen, die älter als einen Monat sind, sollten durch Applikation einer T-Platte auf der medialen Seite der proximalen Tibia behandelt werden (Abb. 20, 21, 22) (White et al., 1982). So können 2 Schrauben in die proximale Epiphyse eingeführt werden, was 


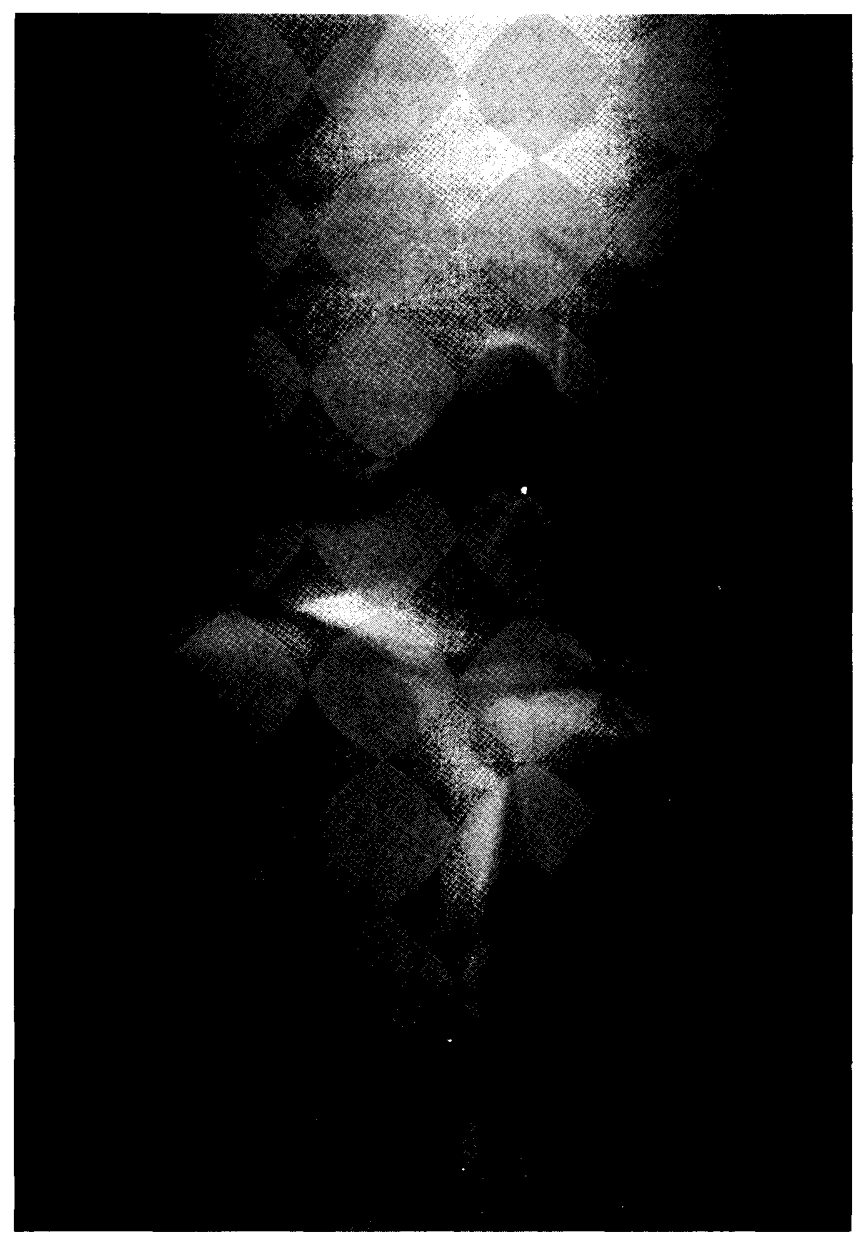

Abb. 19: Kaudokraniales Röntgenbild eines 1 Monate alten QuarterHorse-Fohlens mit einer Salter-Harris-Typus-II-Epiphysenfraktur der proximalen Tibia.

dann eine anatomische Reduktion der Fraktur zur Folge hat. Zusätzlich können Zugschrauben den metaphysealen Teil einer Salter-Harris-Typus-II-Fraktur unter Kompression bringen. Eine weitere Möglichkeit der Behandlung ist das Anbringen von Zugschrauben durch die Metaphyse von medial in den lateralen Anteil der Epiphysenfraktur (Wagner et al., 1984). Im allgemeinen kann bei diesen Frakturen eine günstige Prognose gestellt werden.

Abrißfrakturen der Crista tibialis können durch eine beliebige Art von Zuggurtung in Kombination mit Zugschrauben an die Tibia fixiert werden.

Frakturen der distalen Epiphyse treten selten auf, sind aber sehr schwierig zu behandeln, da der architektonische Aufbau der distalen Epiphyse sehr kompliziert ist. Bei Frakturen in dieser Gegend ist meistens das Talokruralgelenk beteiligt, da die Epiphyse relativ dünn ist. Dies entspricht einer Salter-Harris-Fraktur des Typus III oder IV (DeBowes et al., 1983). Gelenkrekonstruktion und Osteosynthese sind in diesen Frakturen unumgänglich.

\section{Komplikationen}

Die meisten Komplikationen treten im Zusammenhang mit postoperativer Infektion, Gelenksinkongruenz und lokalen Wachstumsstörungen auf (Salter, 1980).
Sekundäre Osteomyelitis, als Folge einer offenen Fraktur oder nach chirurgischer Reduktion einer geschlossenen Fraktur, ist schwieriger zu behandeln und hat beim Jungtier, da der Knochen weicher ist und der Zerstörung nur schwachen Widerstand leisten kann, schlimmere Folgen als beim ausgewachsenen Tier (Salter, 1980; Watkins und Auer, 1984). Im weiteren ist eine Zerstörung der Epiphyse in den meisten Fällen mit einer Wachstumsstörung verbunden, was Verkürzung der Knochen zur Folge hat und dadurch einen Wettkampfeinsatz in Frage stellt (Watkins und Auer, 1984). Es ist wichtig, die Fohlen vor einer Operation unter wirksamen Antibiotikaschutz zu stellen und, falls eine Infektion auftritt, die Infektionserreger mittels mikrobiologischer Labormethoden zu identifizieren. Durch Resistenztestbestimmung der Erreger können die wirksamen Antibiotika bestimmt werden (Aron, 1979).

Frakturen mit intraartikulären Fragmenten müssen unbedingt anatomisch reduziert werden, um einer degenerativen Gelenkserkrankung vorzubeugen. Dabei ist die durch Knorpelabbauprozesse entstehende Synovitis maßgeblich beteiligt (Auer und Fackelman, 1980; Newton, 1981).

Lokale Wachstumsstörungen können zu einem späteren Zeitpunkt durch eine Keil-, Dom- oder Stufenosteotomie

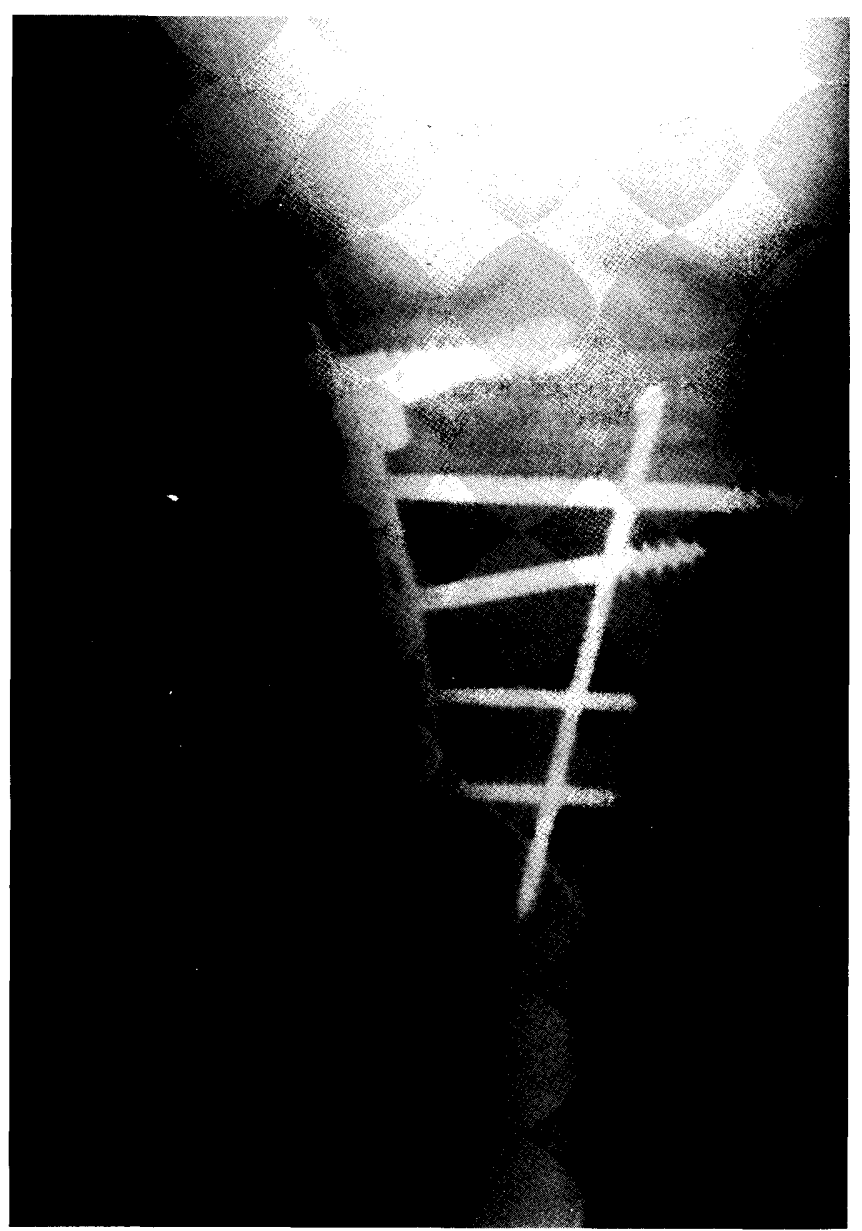

Abb. 20: Kaudokraniales Röntgenbild des gleichen Fohlens wie in Abb. 19 nach der Fixation der Fraktur. Mittels einer T-Platte und Schrauben wurde die Fraktur fixiert. Zwei Zugschrauben wurden in das metaphyseale Fragment implantiert. Zusätzlich wurde ein Steinmann-Nagel von kranial durch die Epiphyse in die Metaphyse eingetrieben. Man beachte die gute Reduktion der Fraktur. 


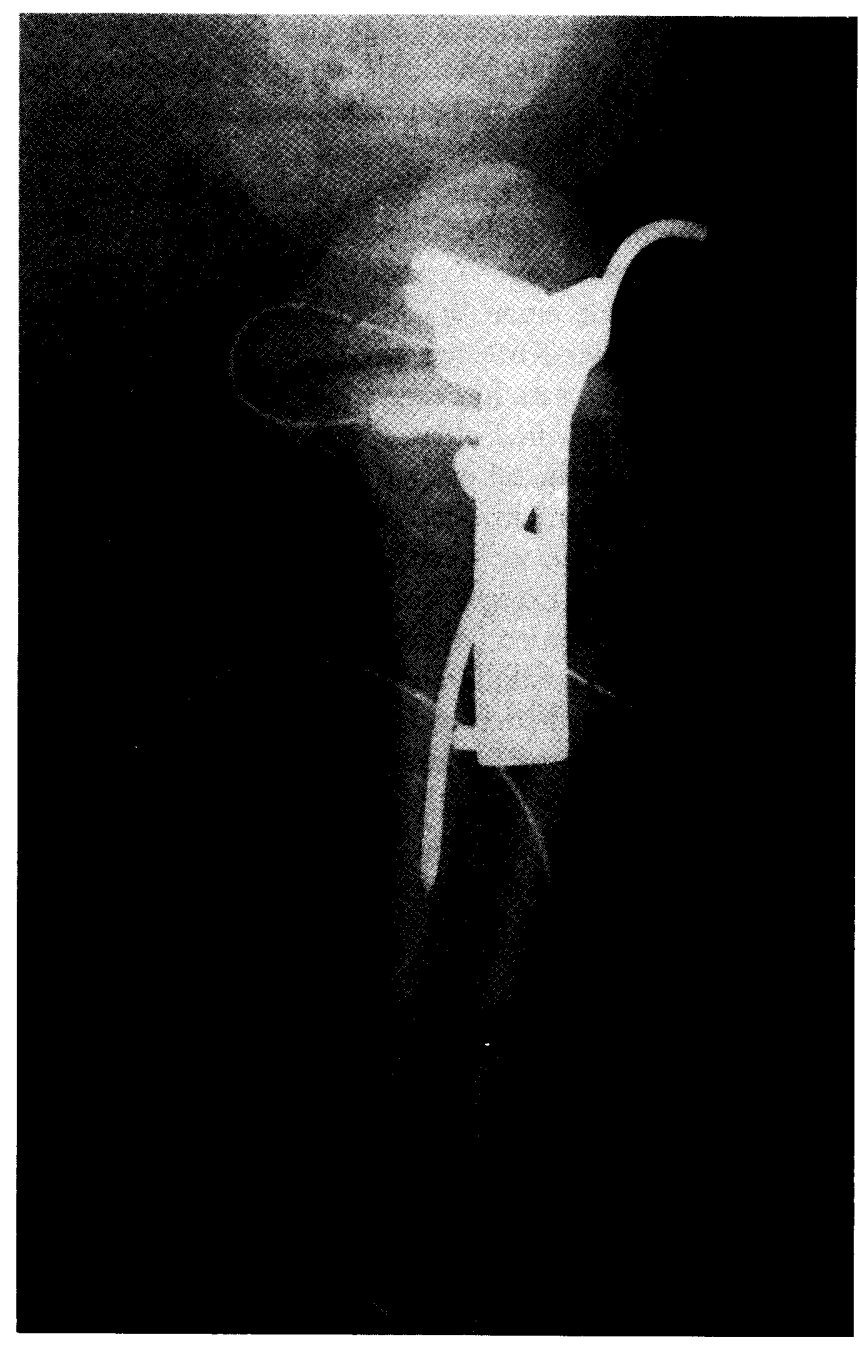

Abb. 21: Lm-Röntgenbild der proximalen Tibia nach Fixation. Die Lokalisation des Steinmann-Nagels ist gut ersichtlich. Ein Saugdrain mit einer röntgenologisch sichtbaren Markierung ist ersichtlich. Dieser Drain wurde 3 Tage nach der Operation gezogen.

korrigiert werden (Auer et al., eingesandt). Doch sind damit weitere Kosten und Risiken verbunden. Deshalb ist es wichtig, diesen Wachstumsstörungen vorzubeugen. Sollte sich die Wachstumsplatte auf der ganzen Länge verfrüht schließen, so resultiert ein kürzerer Knochen aus dieser Wachstumsstörung. Bei älteren Fohlen mit relativ wenig Residualwachstum in der Epiphysenfuge sind diese Verkürzungen minimal und fallen optisch nicht auf, da sie durch Änderung der Gelenkwinkel korrigiert werden können (O’Brien, 1971). Bei jüngeren Fohlen können jedoch starke Verkürzungen auftreten, die optisch leicht erkennbar sind. Die Wahrscheinlichkeit der Entwicklung von Wachstumsstörungen nach Epiphysenfrakturen wird beim Hund und Menschen zwischen 7 und $50 \%$ beschrieben (Marretta und Schrader, 1983; Weber, 1980; Lombardo und Harvey, 1977). Die Salter-Harris-Klassifikation sollte für die Vorhersage einer solchen Entwicklung nützlich sein (Salter, 1980), doch scheint der Verschiebungsgrad der einzelnen Fragmente auch eine Rolle zu spielen (Lombardo und Harvey, 1977). In einer retrospektiven Studie von 72 Fohlen mit Epiphysenfrakturen konnten Embertson et al. (1986 b) keinen signifi- kanten Unterschied zwischen den verschiedenen SalterHarris-Klassifikationstypen finden.

Zusammenbruch der Fixation ist eine Komplikation, die vor allem bei älteren Fohlen auftritt (Turner, 1982 c). Die Osteosynthese hat den Vorteil, daß die Patienten eine bessere Chance für eine athletische Karriere besitzen. Tritt jedoch ein Zusammenbruch der Osteosynthese auf, so muß in den meisten Fällen das Tier aus humanen Gründen euthanasiert werden (Turner, 1982 c). Die Kosten für eine Behandlung mittels Osteosynthese sind erheblich und müssen in Relation zum Wert des Tieres gestellt werden.

Es ist wichtig, daß der Chirurg, welcher diese Operationen vornimmt, Erfahrungen mit der Fixation von Röhrenbeinen hat. Sollte dies nicht der Fall sein, so sind Komplikationen eher zu erwarten (Turner, 1981).

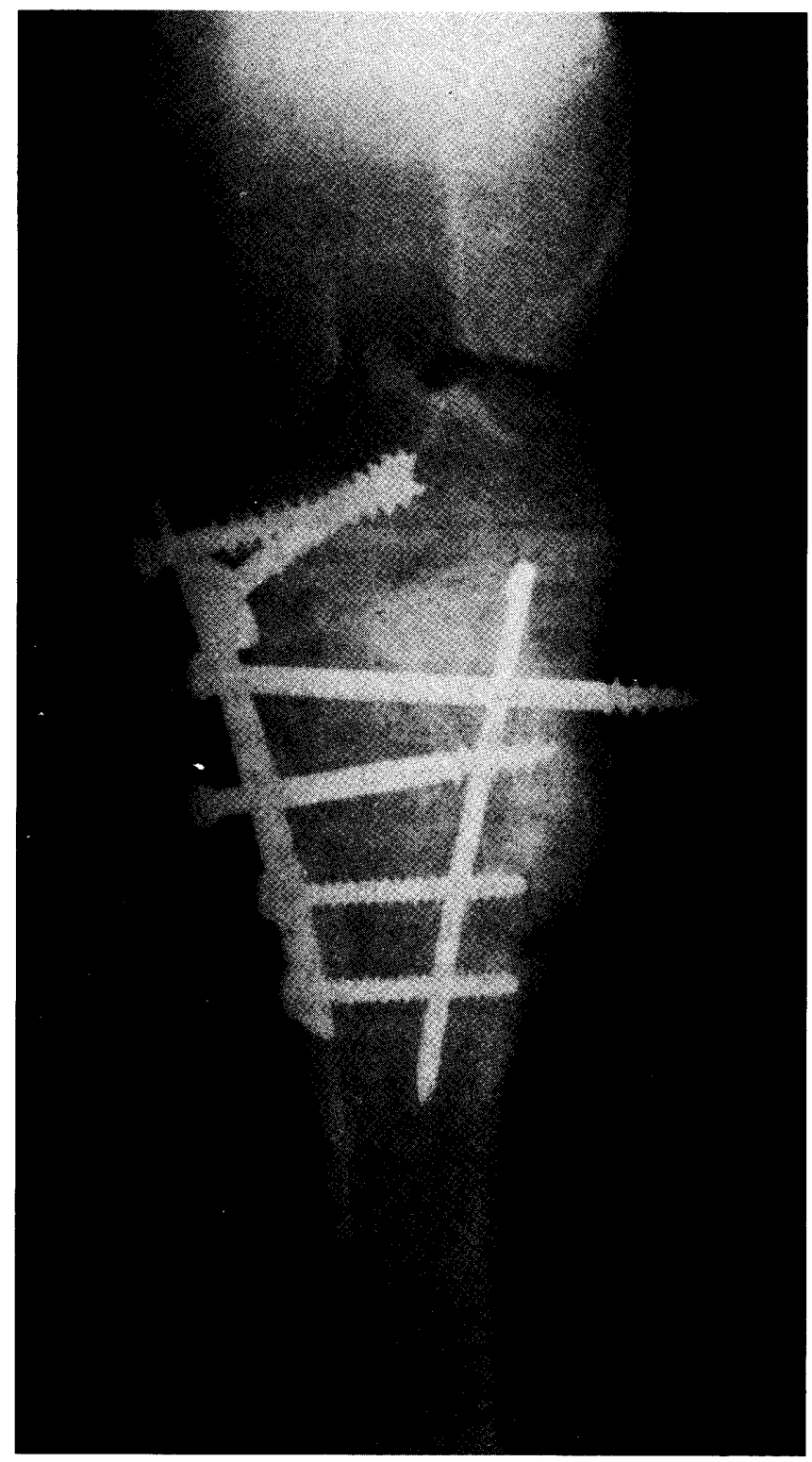

Abb. 22: Kaudokraniales Röntgenbild des gleichen Fohlens wie in Abb. 19 bis 21 nach Verheilung der Fraktur. 3 Monate nach der Operation wurden die Implantate entfernt. Man beachte, daß sich 3 der obersten 4 Schrauben etwas gelöst hatten. Dennoch verheilte die Fraktur komplikationslos, und das Fohlen verzeichnete keine Lahmheit. 


\section{Literatur}

Aron, D. N. (1979): Pathogenesis, diagnosis, and management of osteomyelitis in small animals. Compend. Contin. Educ. Pract. Vet. 1, $824-830$.

Auer, J. A., und Fackelman, G.E. (1980): Treatment of degenerative joint disease. A review and commentary. Vet. Surg. 10, 80-89.

Auer, J.A., und Martens, J.R. (1980): Angular limb deformities in young foals. Proc. 26th. Ann. Meet. Amer. Assoc. Equine Pract. 26, 81-96.

Auer, J. A., Bramlage, L. R., und Watkins, J.P. (1986): Corrective osteotomies in the horse. Equine Vet. J. (eingesandt).

Braden, T.D. (1981): Epiphyseal injuries. In: Pathophysiology in small animal surgery. Ed. M. J. Bojrab. Lea \& Febiger, Philadelphia. 791-801.

Bramlage, L. R. (1982): Longbone fractures. Vet. Clin. N. Amer. Large Anim. Pract. 5, 285-310.

Bramlage, L.R. (1983): Current concepts of emergency first aid treatment and transportation of equine patients. Compend. Contin. Educ. Pract. Vet. 5, 126-135.

Brashear, H.R. (1959): Epiphyseal fractures. J. Bone Joint Surg. 41 A, 1055-1064

Campbell, C. H., Grisiola, A., und Zancoato, G. (1959): The effects produced in the cartilaginous epiphyseal plate of immature dogs by experimental surgical traumata. J. Bone Joint Surg. 41 A, 1221-1242.

Colahan, P. T., und Meagher, D. M. (1979): Repair of comminuted fractures of the proximal ulna and olecranon in young horses using tension band plating. Vet. Surg. 8, 105-111.

Daly, W.R. (1978): Femoral head and neck fractures in the dog and cat: A review of 115 cases. Vet. Surg. 7, 29-30.

DeBowes, R. M., Grant, B.D., und Modransky, P.D. (1983): Lag screw stabilization of Salter type IV femoral fracture in a young horse. J. Amer. Vet. Med. Assoc. 182, 1123-1125.
Donecker, J.M., Bramlage, L.R., und Gabel, A.A. (1984): Retrospective analysis of 29 fractures of the olecranon process of the equine ulna. J. Amer. Vet. Med. Assoc. 185, 183-189.

Embertson, R. M., Bramlage, L. R., Herring, D. S., und Gabel, A. A. (1986 a): Physeal fractures in the horse: I Classification and incidence. Vet. Surg. 15, 223-229.

Embertson, R. M., Bramlage, L. R., und Gabel, A.A. (1986 b): Physeal fractures in the horse: II Management and outcome. Vet. Surg. 15, 230-236.

Eulner, W.P. (1974): Epiphysenfrakturen in den Extremitäten des Pferdes. Dissertation der Tierärztlichen Hochschule Hannover.

Fackelman, G.E. (1984): Deformities of the appendicular skeleton. In: The practice of large animal surgery. Ed. P. B. Jennings. W. B. Saunders Company, Philadelphia. 950-982.

Fackelman, G. E., und Nunamaker, D.M. (1982): Manual of internal fixation in the Horse. Springer Verlag, Berlin, Heidelberg.

Fretz, P. B., und McIlwraith, C. W. (1982): Wedge osteotomy as a treatment for angular limb deformities of the fetlock in horses. J. Amer. Vet. Med. Assoc. 182, 245-250.

Gaines, J.D., und Auer, J.A. (1983): Case report: Treatment of a SalterHarris Type III epiphyseal fracture in a young horse. Compend. Contin. Educ. Pract. Vet. 5, 102-106.

Ham, A. W., und Cormack, D. H. (1979): Histology. J. P. Lipincott Company, Philadelphia. 422-449.

Hamilton, G.F., Turner, A.S., Ferguson, J. G., und Pharr, J.W. (1978): Slipped capital femoral epiphysis in calves. J. Amer. Vet. Med. Assoc. $172,1318-1322$.

Leitch, $M$. (1977): A review of treatment of tuber scapulae fractures in the horse. J. Equine Med. Surg. 1, 234-240.

Llewellyn, H.R. (1976): Growth plate injuries: Diagnosis, prognosis and treatment. J. A. A. H. A. $12,77-82$.

\section{CAPSOLIN}

\section{die stark hyperämisierende Salbe}

Zusammensetzung

$00 \mathrm{~g}$ Salbe enthalten: Oleoresina Capsici Camphora

Oleum Terebinthinae rectif.

Oleum Eucalypti .

Oleum Lavandulae

$1,200 \mathrm{~g}$

$5,250 \mathrm{~g}$

$9,750 \mathrm{~g}$

$2,500 \mathrm{~g}$

$0,623 \mathrm{~g}$

Anwendungs- Chronische Gelenk- und Sehnenscheidenentzündungen, gebiete

heumatische Erkrankungen, Aktivierung von Abszen, und Hämatomen, Erweichung von chronischen Mastitiden (Steineutern)

Dosierungs- Capsolin wird in ausreichender Menge auf das zu beanleitung handelnde Gebiet aufgetragen und gut einmassiert. Die leitung und Art

der Anwendung Behandlung wird 2-3mal täglich wiederholt.

\section{Wartezeit Eßbares Gewebe 3 Tage, Milch 3 Tage}

Eigen- Capsolin ist eine Salbe mit stark hyperämisierendem Effekt schaften und langanhaltender Tiefenwirkung ohne die Gefahr einer Hautschädigung.

Besondere Nach dem Einmassieren sind die Hände mit warmem Hinweise Wasser und Seife gründlich zu säubern. Die Salbe verursacht auf Schleimhäuten oder Wunden heftige Reizerscheinungen.

Darreichungs- Tube mit $100 \mathrm{~g}$ Salbe

form und Tube mit $450 \mathrm{~g}$ Salbe

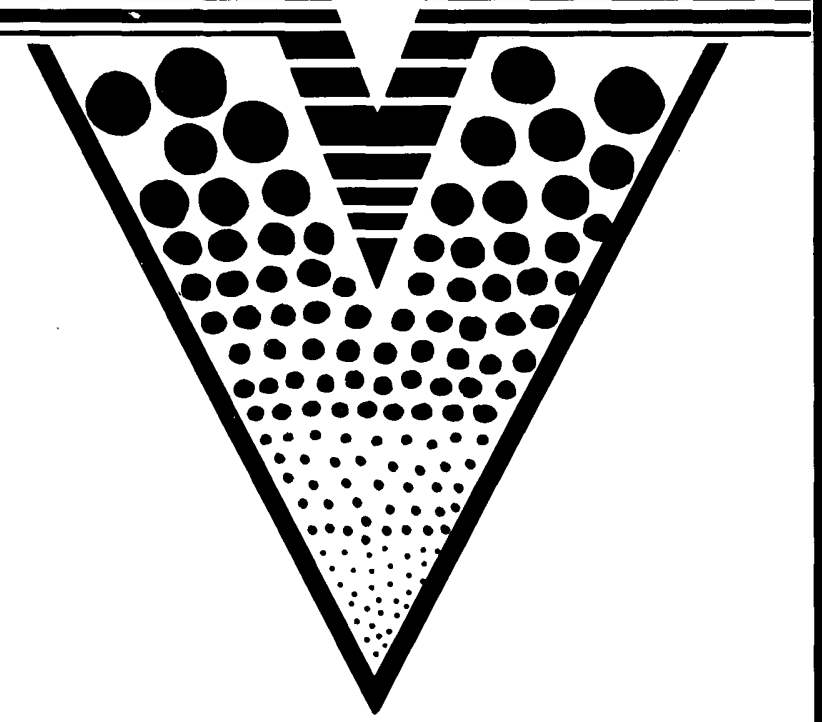

PARKE-DAVIS

Parke, Davis \& Company, Berlin Postanschrift: Postfach 56 20, 7800 Freiburg 
Lombardro, S. J., und Harvey, J. P. (1977): Fractures of the distal femoral epiphyses. Factors influencing prognosis: A review of thirty-four cases. J. Bone Joint Surg. 59 A, 742-751.

Marretta, S. M., und Schrader, S. C. (1983): Physeal injuries in the dog: A review of 135 cases. J. Amer. Vet. Med. Assoc. 182, 708-710.

Monin, T. (1978): Repair of physeal fractures of the tuber olecranon in the horse using a tension band method. J. Amer. Vet. Med. Assoc. 172, 287-290.

Müller, M.E., Allgöwer, M., Schneider, R., und Willenegger, H. (1979): Manual of internal fixation. 2nd ed. Springer Verlag, Berlin, Heidelberg.

Newton, C. D., Nunamaker, D.M., und Dickinson, C. R. (1975): Surgical management of radial physeal growth disturbance in dogs. J. Amer. Vet. Med. Assoc. 167, 1011-1018.

Newton, C.D. (1981): Traumatic arthritis in the dog. In: Pathophysiology in small animal surgery. Ed. M. J. Bojrab. Lea \& Febiger, Philadelphia, $568-570$.

Nickel, R., Schummer, A., und Seiferle, E. (1968): Lehrbuch der Anatomie der Haustiere. Band I. Dritte Aufl., 11-22.

O'Brien, J.R. (1971): Developmental deformities due to arrested epiphyseal growth. Vet. Clin. N. Amer. 1, 441-454.

Pollen, A. G. (1979): Fractures involving the epiphyseal plate. Reconstr. Surg. Traumatol. 17, 25-39.

Regazzoni, P., Ruedi, T., Winquist, R., und Allgöwer, M. (1985): The dynamic hip screw implant system. Springer Verlag, Berlin, Heidelberg.

Rogers, L.F. (1970): The radiography of epiphyseal injuries. Radiology 96, 289-299.

Salter, R. B., und Harris, W. R. (1963): Injuries involving the epiphyseal plate. J. Bone Joint Surg. 45 A, 587-622.

Salter, R. B. (1980): Birth and pediatric fractures. In: Fracture treatment and healing. Ed. R. B. Heppenstall. W. B. Saunders Company, Philadelphia. 198-234.

Schmidt, G. (1960): Epiphysen und Apophysen in der röntgenologischen Darstellung an den Vorder- und Hinterextremitäten der Fohlen. Dissertation der Tierärztlichen Hochschule Hannover.

Stover, S. M., und Rick, M. C. (1985): Ulnar subluxation following repair of a fractured radius in a foal. Vet. Surg. $14,27-31$.

Tachidjian, M. O. (1972): Fractures and dislocations. In: Pediatric orthopedics. Ed M. O. Tachidjian. W. B. Saunders Company, Philadelphia. 1532-1754.

Turner, A.S., Milne, D. W., und Gabel, A.A. (1976): Use of the dynamic compression plate for treatment of equine long bone fractures. J. Amer. Vet. Med. Assoc. 168, 309-315.
Turner, A. S., Milne, D. W., Hohn, R. B., und Rouse, G. P. (1979): Surgical repair of fractured capital femoral epiphyses in three foals. J. Amer. Vet. Med. Assoc. 175, 1198-1202.

Turner, A.S. (1981): Long bone fractures in horses. Part I. Initial management. Compend. Contin. Educ. Pract. Vet. 3, 347-354.

Turner, A. S. (1982 a): Long bone fractures in horses. Part II. Intraoperative techniques. Compend. Contin. Educ. Pract. Vet. 4, 196-202.

Turner, A. S. (1982 b): Long bone fractures in horses. Part. III. Postoperative management. Compend. Contin. Educ. Pract. Vet. 4, 254-257.

Turner, A. S. (1982 c): Fractures of specific bones. In: Equine medicine and surgery. Eds R. A. Mansmann and E. S. McAllister. American Veterinary Publications Inc., Santa Barbara. 997-1020.

Turner, A.S. (1984): Large animal orthopedics. In: The practice of large animal surgery. Ed P. B. Jennings. W. B. Saunders Company, Philadelphia. 768-949.

Wagner, D. C., DeBowes, R.M., Grant, B.D., Kaneps, A.J., und Watrous, $B . J$. (1984): Cancellous bone screws for repair of proximal growth plate fractures of the tibia in foals. J. Amer. Vet. Med. Assoc. 184, 688-691.

Wagner, P. C., Watrous, B. J., Shires, G. M., und Riebold, T. W. (1985): Resection of the supraglenoid tubercle of the scapula in a colt. Compend. Contin. Educ. Pract. Vet. 7, 536-540.

Watkins, J.P., und Auer, J.A. (1984): Physeal injuries. Compend. Contin. Educ. Pract. Vet. 6, 226-235.

Watkins, J.P., Auer, J.A., und Taylor, T.S. (1985): Crosspin fixation of fractures of the proximal tibia in three foals. Vet. Surg. 14, 153-159.

Weber, B.G. (1980): Facture healing in the growing bone and in the mature skeleton. In: Treatment of fractures in children and adolescents. Ed. B. G. Weber. Springer Verlag, New York. 20-57.

White II, N. A., Blackwell, R. B., und Hoffman, P.E. (1982): Use of a bone plate for repair of proximal physeal fractures of the tibia in two foals. J. Amer. Vet. Med. Assoc. 181, 252-254.

J. A. Auer, DMV, MS

Diplomate American College of Veterinary Surgeons

Associate Professor of Veterinary Medicine and Surgery

Large Animal Veterinary Teaching Hospital

College of Veterinary Medicine

Texas A E $M$ University

College Station, Texas, 77843

USA

\section{Kurzreferat}

\section{Endometritistherapie bei Stuten}

\section{(Therapy of Endometritis in Mares)}

\section{G. Bennett (1986)}

JAVMA 188, 1390-1391

Die bakteriellbedingte Endometritis ist die häufigste Ursache für die Unfruchtbarkeit bei Stuten. Bei geeigneter Therapie kann jedoch eine Trächtigkeitsrate von $70-80$ Prozent erreicht werden.
Pathogene Bakterien, durch Koitus oder Geburt in den Uterus eingeschleppt, werden bei gesunden Stuten in der Regel innerhalb weniger Stunden eliminiert. Bei Tieren, die für Endometritis anfällig sind, nisten sich die Bakterien ein und erzeugen eine für eine Trächtigkeit unvorteilhafte Umgebung.

Die Konzentration von neutrophilen Granulozyten und Immunglobulinen im Uterus anfälliger Stuten unterscheidet sich nicht von der gesunder Tiere. Dagegen findet man bei resistenten Stuten eine größere phagozytotische und bakterizide Aktivität der neutrophilen Granulozyten. Die Fähigkeit, Infektionen abzuwehren, ist im Östrus größer als während des Diöstrus. Nach Erstellen von Antibiogrammen und Bakterienkulturen ist eine Antibiotikatherapie häufig empfehlenswert. Die intrauterine Behandlung wird der systemischen Therapie vorgezogen. Ihr Vorteil liegt in der wesentlich höheren Antibiotikakonzentration 